\title{
Regionale Unterschiede der Verteilung von Personen mit unternehmerischem Persönlichkeitsprofil in Deutschland - ein Überblick
}

\author{
Michael Fritsch ${ }^{1}$ (D) - Martin Obschonka ${ }^{2}$ Michael Wyrwich ${ }^{1} \cdot$ Samuel D. Gosling ${ }^{3} \cdot$ Peter J. Rentfrow $^{4}$. \\ Jeff Potter ${ }^{5}$
}

Eingegangen: 9. Juni 2017 / Angenommen: 10. Januar 2018 / Online publiziert: 16. Januar 2018

(c) Springer-Verlag GmbH Deutschland, ein Teil von Springer Nature 2018

\section{Zusammenfassung}

Neuere Forschungen brachten deutliche Hinweise auf regional unterschiedliche Ausprägungen bestimmter Persönlichkeitsmerkmale in der Bevölkerung. Solche regionalen Unterschiede bei prägenden Verhaltenseigenschaften einer Bevölkerung können wesentlich zur Erklärung einer regionalen Entwicklung beitragen. Wir geben einen Überblick über regionale Unterschiede von unternehmerischen Persönlichkeitsmerkmalen in der Bevölkerung in Deutschland. Es zeigt sich eine Reihe von hochsignifikanten regionalen Verschiedenheiten der Persönlichkeitsprofile, allerdings erweist sich die Effektstärke als relativ gering. Insgesamt deuten die Befunde darauf hin, dass die regionalen Unterschiede sowohl auf selektive Migration als auch auf Sozialisationseffekte zurückgeführt werden können.

Schlüsselwörter Persönlichkeitsmerkmale $\cdot$ Unternehmerische Persönlichkeit · Big Five · Entrepreneurship · Regionale Kultur

Prof. Dr. Michael Fritsch

m.fritsch@uni-jena.de

PD Dr. Martin Obschonka

martin.obschonka@qut.edu.au

PD Dr. Michael Wyrwich

m.wyrwich@uni-jena.de

Prof. Samuel D. Gosling, PhD.

samg@austin.utexas.edu

Dr. Peter J. Rentfrow

pjr39@cam.ac.uk

Jeff Potter

jeff@thebigfiveproject.com

1 Lehrstuhl für Unternehmensentwicklung, Innovation und wirtschaftlichen Wandel, Friedrich-Schiller-Universität Jena, Carl-Zeiss-Straße 3, 07743 Jena, Deutschland

2 QUT Business School, Queensland University of Technology, GPO Box 2434, QLD 4001 Brisbane, Australien

3 Department of Psychology, University of Texas, 1 University Station, Austin, TX 78712, USA

4 Department of Psychology, University of Cambridge, Downing Site, Cambridge, CB 2 3EB, Großbritannien

5 Atof Inc., 17 Magazine Street, Cambridge, MA 02139, USA 


\title{
Regional differences of people with an entrepreneurial personality structure in Germany - An overview
}

\begin{abstract}
Recent research has found clear indications of regional differences in the personality traits of the population. Such regional differences may significantly contribute to explaining regional development. We provide an overview of regional differences in entrepreneurial personality traits among the population in Germany. There are a number of highly significant regional differences but the strength of the effects is relatively small. The empirical evidence suggests that the regional differences found are due to selective migration and also to socialization.
\end{abstract}

Keywords Personality traits $\cdot$ Entrepreneurial personality $\cdot$ Big Five $\cdot$ Entrepreneurship $\cdot$ Regional cultures

\section{Regionale Unterschiede von Persönlichkeitsmerkmalen (Einführung)}

Zur Erklärung von regionalen Unterschieden der ökonomischen Struktur und der wirtschaftlichen Entwicklung werden nicht selten - neben anderen Faktoren - unterschiedliche Mentalitäten und ,landsmannschaftliche Besonderheiten' der Bevölkerung angeführt, die das individuelle Verhalten prägen (Hartke 1959; Miegel 1991). Dies schlägt sich dann unter anderem in gängigen Stereotypen nieder wie etwa dem des „strebsam-findigen Schwaben“ (Siart/Lang 2016), des „lebenslustigen Rheinländers“ oder des ,unterkühlten Norddeutschen“. Die empirische Grundlage für solche Stereotype ist allerdings in aller Regel bruchstückhaft und nur wenig belastbar. Tatsächlich mangelte es bisher an soliden Informationen über regionale Unterschiede von Persönlichkeitsmerkmalen der Bevölkerung, aus denen man auf regionale Mentalitäten und entsprechende wirtschaftliche Folgen schließen könnte. ${ }^{1}$

Der vorliegende Beitrag will zur Schließung dieser Lücke beitragen. Auf der Grundlage von neuerdings verfügbaren Daten über die Big-Five-Persönlichkeitsmerkmale (vgl. Kapitel 2.1) der Bevölkerung konstruieren wir das Profil einer unternehmerischen Persönlichkeit und geben einen Überblick über deren regionale Verteilung in Deutschland. Wie in Studien für die USA (Obschonka/SchmittRodermund/Silbereisen et al. 2013; Stuetzer/Audretsch/ Obschonka et al. 2017) und für Großbritannien (Stuetzer/ Obschonka/Audretsch et al. 2016) ${ }^{2}$ zeigen sich auch in Deutschland einige deutliche regionale Unterschiede, von denen vermutet werden kann, dass sie für die regionale Entwicklungsdynamik - insbesondere für das regionale Niveau und den Wachstumsbeitrag durch unternehmerische Selbstständigkeit - von Bedeutung sind. Unsere Ergebnisse stellen einen ersten, überwiegend deskriptiven Schritt der

\footnotetext{
${ }^{1}$ Eine der wenigen Ausnahmen ist die Studie von Falck/Heblich/ Lameli et al. (2012), die auf Informationen über regionale Dialekte beruht.

$2 \mathrm{Zu}$ den Studien vgl. Kapitel 2.1.
}

Auswertung dieser neuerdings für Deutschland verfügbaren Daten dar. Die Ursachen und die Folgen der gefundenen regionalen Unterschiede müssen in weiteren Arbeiten näher analysiert werden.

Nachfolgend beschreiben wir die Annahme einer unternehmerischen Persönlichkeitsstruktur sowie deren empirische Operationalisierung (Kapitel 2). In Kapitel 3 wird zunächst eine psychologische Landkarte Deutschlands erstellt, die einen Überblick über die regionale Verteilung von Menschen mit unternehmerischem Persönlichkeitsprofil in der Bevölkerung in den deutschen Raumordnungsregionen gibt (Kapitel 3.1). Daran anschließend betrachten wir Unterschiede zwischen Regionen mit unterschiedlichem Verdichtungsgrad (Kapitel 3.2), zwischen Ost- und Westdeutschland (Kapitel 3.3) sowie zwischen Nord- und Süddeutschland (Kapitel 3.4). Schließlich werden die wesentlichen Ergebnisse zusammengefasst und Schlussfolgerungen für weitere Forschungen gezogen (Kapitel 4).

\section{Konzept und empirische Bestimmung der unternehmerischen Persönlichkeit}

\subsection{Die unternehmerische Persönlichkeitsstruktur}

Die wissenschaftliche Literatur zum Thema Unternehmertum fokussierte schon früh auf die Annahme, dass Unternehmer sich von anderen Personen hinsichtlich ihrer Persönlichkeitsstruktur unterscheiden (Schumpeter 1934; Chell 2008). Eine Reihe von empirischen Untersuchungen hat in der Tat gezeigt, dass sich unternehmerisch tätige Personen hinsichtlich ihrer Persönlichkeitsprofile häufig von nichtunternehmerisch tätigen Personen abheben. Dies betrifft etwa Persönlichkeitsmerkmale wie die Fähigkeit zum Tragen von Risiko, Kreativität, Selbstvertrauen, proaktive Handlungsbereitschaft, interne Kontrollüberzeugung, Eigenverantwortlichkeit, Leistungsorientierung und Stresstoleranz, die bei unternehmerisch tätigen Personen im Durchschnitt besonders stark ausgeprägt sind und allgemein als förderlich für die erfolgreiche Gründung eines Unternehmens an- 
gesehen werden (Rauch/Frese 2007; Zhao/Seibert/Lumpkin 2010; Caliendo/Fossen/Kritikos 2014).

Entsprechende Untersuchungen lassen darauf schließen, dass auch weiter gefasste Persönlichkeitsmerkmale wie die „Big Five“ (McCrae/Costa 1999; Zhao/Seibert/Lumpkin 2010; Caliendo/Fossen/Kritikos 2014) mit Unternehmertum in Zusammenhang stehen. Die Big Five sind das führende und am besten validierte Persönlichkeitsmodell zu Traits in der zeitgenössischen psychologischen Forschung (Digman 1990; Benet-Martínez/John 1998). Unter Traits versteht man relativ stabile Persönlichkeitsmerkmale, die eine gewisse Konsistenz im Erleben und Verhalten über die Zeit und über unterschiedliche Situationen hinweg anzeigen (John/Srivastava 1999). Bisherige Ergebnisse deuten darauf hin, dass die Big Five stark genetisch mitbestimmt sind (Loehlin 1992; Shane/Nicolaou/Cherkas et al. 2010) und die Struktur dieser Persönlichkeitsmerkmale im Erwachsenenalter relativ stabil ist. Diese Stabilität der Persönlichkeitsmerkmale schließt allerdings nicht aus, dass sie auch durch Umgebungseinflüsse und normative Veränderungen geprägt sein können (Loehlin/McCrae/Costa et al. 1998; Roberts/Walton/Viechtbauer 2006).

Das Big-Five-Modell basiert auf der empirisch validierten Annahme, dass sich die dispositionale Grundstruktur der Persönlichkeit eines Menschen in nahezu allen Kulturen der Erde mit fünf Merkmalen hinreichend erfassen lässt. Bei diesen Merkmalen handelt es sich um:

- Offenheit für Erfahrungen

- Extraversion (Außenorientierung und Aktivität)

- Gewissenhaftigkeit

- Neurotizismus (geringe Belastbarkeit, emotionale Labilität)

- Verträglichkeit im Sinne von geringer Konflikt- und Wettbewerbsbereitschaft

Empirische Untersuchungen weisen darauf hin, dass sich die ersten drei dieser Big-Five-Dimensionen, nämlich Offenheit für Erfahrungen, Extraversion und Gewissenhaftigkeit, positiv auf die Gründungsneigung einer Person und ihre unternehmerischen Fähigkeiten auswirken. Demgegenüber scheinen Neurotizismus sowie geringe Konfliktbereitschaft und Harmoniestreben (Verträglichkeit) eher einen negativen Einfluss auf die Gründungsneigung zu haben (vgl. z. B. Zhao/Seibert/Lumpkin 2010; Caliendo/Fossen/Kritikos 2014). Interessanterweise passt dieses intraindividuelle Big-Five-Muster (hohe Offenheit, Extraversion, Gewissenhaftigkeit sowie geringe Verträglichkeit und niedriger Neurotizismus) sowohl zu anekdotischen Belegen hinsichtlich der Persönlichkeitsstruktur besonders erfolgreicher Unternehmer (Isaacson 2011) als auch zu frühen Theorien einer unternehmerischen Persönlichkeit (Schumpeter 1934).
Diese empirisch identifizierten Zusammenhänge zwischen den Big-Five-Persönlichkeitsmerkmalen und erfolgreichem Unternehmertum lassen sich wie folgt plausibilisieren: Offenheit für Erfahrungen begünstigt die Absorption von Wissen sowie insbesondere den Entschluss, die Option der unternehmerischen Selbstständigkeit in die Tat umzusetzen. Auch Außenorientierung begünstigt Lerneffekte; das Erkennen unternehmerischer Gelegenheiten und ist vor allem auch für die Vermarktung erforderlich. Gewissenhaftigkeit fördert das zuverlässige Abarbeiten der anfallenden Aufgaben. Weiterhin erfordert das erfolgreiche Führen eines Unternehmens emotionale Stabilität (geringes $\mathrm{Ma} ß$ an Neurotizismus) sowie Konfliktfähigkeit, Wettbewerbsorientierung und Non-Konformismus (niedriges $\mathrm{Ma} \beta$ an Verträglichkeit) (vgl. auch Obschonka/Schmitt-Rodermund/Silbereisen et al. 2013).

Aufbauend auf diesen Überlegungen geht das Konzept der unternehmerischen Persönlichkeitsstruktur davon aus, dass Menschen mit einem bestimmten Profil eher zu Unternehmertum neigen als andere - was nicht ausschließt, dass auch Menschen mit anderen Profilen Unternehmer werden. Dieses intraindividuelle unternehmerische Profil ist gekennzeichnet durch ein gleichzeitiges Auftreten hoher Offenheit für Erfahrungen, Extraversion und Gewissenhaftigkeit sowie geringem Neurotizismus und geringer Verträglichkeit in der Persönlichkeitsstruktur einer Person. Es handelt sich damit um eine personenorientierte Sichtweise (Magnusson/ Törestad 1993). Das Konzept der unternehmerischen Persönlichkeitsstruktur bezieht die Gesamtheit und Konfiguration der zentralen Traits einer Person (z. B. die Big Five) in die Analyse ein, da die Persönlichkeit eher über die Konstellation der zentralen Traits einer Person als über einen isolierten Trait allein (wie etwa in der variablenorientierten Persönlichkeitsforschung) beschreibbar ist (vgl. ausführlich Obschonka/Stuetzer 2017). Die individuelle Passung zu einem unternehmerischen Referenzprofil, wie von Obschonka, Schmitt-Rodermund, Silbereisen et al. (2013) vorgeschlagen, erfasst daher den grundlegenden unternehmerischen Charakter einer Person in ihrer Gesamtheit.

Die empirische Forschung zu diesem intraindividuellen unternehmerischen Persönlichkeitsprofil liefert deutliche Hinweise für die Validität dieses Profils. Studien auf der Individualebene zeigen, dass dieses Profil unternehmerische Aktivität - sowohl die Absicht zur Gründung eines Unternehmens als auch die unternehmerische Motivation -, unternehmerische Kompetenz, Leidenschaft und unternehmerische Selbstidentität erwarten lässt (für eine Übersicht vgl. Obschonka/Stuetzer 2017).

Auch auf der Ebene von Regionen lieferte dieses Profil als Merkmal der regionalen Bevölkerung bislang bemerkenswert konsistente Befunde über verschiedene Länder und Konzeptualisierungen von Unternehmertum hinweg. Untersuchungen für die USA und für Großbritannien zei- 
gen, dass dieses Profil zuverlässig mit dem Niveau regionaler unternehmerischer Aktivität korreliert (Obschonka/ Stützer/Gosling et al. 2015). Regionen, in denen das Persönlichkeitsprofil der Bevölkerung stärker unternehmerisch geprägt ist, zeigten während der schweren Weltwirtschaftskrise der Jahre 2008/2009 ein höheres Maß an ökonomischer Resilienz (Obschonka/Stuetzer/Audretsch et al. 2016). Zudem realisieren diese Regionen ein höheres Maß an Wachstum (z. B. Beschäftigungswachstum) (Stuetzer/ Audretsch/Obschonka et al. 2017). Auch konnte gezeigt werden, dass insbesondere solche Regionen eine relativ hohe Gründungsrate aufweisen, die durch eine Kombination von vielen Einwohnern mit stark ausgeprägten unternehmerischen Persönlichkeitsmerkmalen und förderlichen Rahmenbedingungen, wie etwa einem hohen Bildungsstand der Bevölkerung, gekennzeichnet sind (Obschonka/ Schmitt-Rodermund/Silbereisen et al. 2013; Obschonka/ Stuetzer/Gosling et al. 2015). Dieses Muster wird in einigen prominenten Studien als entscheidend für langfristige wirtschaftliche Prosperität angesehen (Saxenian 1996; Florida 2002), ohne dass dies bislang genauer nachgewiesen werden konnte bzw. wurde.

Andere Studien befassen sich mit historischen Einflüssen auf die regionalen Unterschiede in der Ausprägung unternehmerischer Persönlichkeitsprofile der Bevölkerung. So weist die Untersuchung von Audretsch, Obschonka, Gosling et al. (2017) darauf hin, dass die historische Industriestruktur, aber auch historische Migrationsmuster eine entscheidende Rolle spielen. In diesem Sinne fanden Stuetzer, Obschonka, Audretsch et al. (2016) heraus, dass eine Konzentration von Großindustrien sowie die damit verbundenen nichtunternehmerischen Arbeitsanforderungen und lokalen Kulturen einen negativen Effekt auf die Ausprägung unternehmerischer Persönlichkeitsmerkmale der heutigen regionalen Bevölkerung haben. Zudem vermuten die Autoren systematische Migrationsmuster, wie die Abwanderung von unternehmerisch eingestellten Personen aus alten Industrieregionen, in denen Unternehmertum nur schwach ausgeprägt ist.

Die Entwicklung der regionalen Industriestruktur, insbesondere die Herausbildung großer Betriebe mit standardisierter Produktion, und selektive Migration können also zu einem Wandel der Persönlichkeitsmerkmale der regionalen Bevölkerung führen. Auf diese Weise ließe sich etwa erklären, wieso in einstmaligen Gründungsregionen (z. B. dem Rust Belt in den USA) der Wille und die Fähigkeit zu unternehmerischer Selbstständigkeit im Laufe der Zeit abgenommen haben (vgl. Sorenson 2017). Noch unklar ist, warum in bestimmten Regionen, wie allgemein in Großstädten im Vergleich zu ländlichen Gebieten (Obschonka/Schmitt-Rodermund/Silbereisen et al. 2013), höhere Werte für die unternehmerische Persönlichkeit zu finden sind. Wahrscheinlich ist auch hier ein Mix aus Selektion (z. B. systematischer
$\mathrm{Zu}$ - und Wegzug) sowie Sozialisation (Prägung der lokalen Mentalität durch formelle und informelle Institutionen in der Region) für diese räumlichen Muster mitverantwortlich. Ausgehend von psychologischen Theorien der Entstehung und Persistenz regionaler Persönlichkeitsunterschiede (z. B. Rentfrow/Gosling/Potter 2008) lässt sich vermuten, dass die Persönlichkeitsstruktur der regionalen Bevölkerung zumindest über die Dauer von ein, zwei Generationen relativ stabil ist. Mit anderen Worten: Hinter der empirisch häufig zu beobachtenden Persistenz regionaler Unterschiede von unternehmerischer Aktivität (Fritsch/Wyrwich 2014; Fritsch/Wyrwich 2017) könnte die Persistenz der regionalen Unterschiede mit unternehmerischen Persönlichkeiten stehen.

\subsection{Datengrundlage}

Wir analysierten Daten aus der von Jeff Potter und Samuel D. Gosling geleiteten weltweiten Big-Five-Studie, in deren Rahmen seit dem Jahr 2000 über das Internet anonymisiert Daten zur Persönlichkeit erhoben werden. ${ }^{3}$ Die Website des Projektes kann prominent über einschlägige Online-Suchmaschinen gefunden werden und bietet Besuchern die Teilnahme an einer Persönlichkeitsanalyse mittels eines wissenschaftlich fundierten Verfahrens an. Als Instrument zur Ermittlung der Persönlichkeitsmerkmale kommt die deutsche Version des Big Five Inventory (BFI; John/ Srivastava 1999) zum Einsatz, das die Big-Five-Merkmale auf der Grundlage von 44 Items (fünfstufige Likertskala) valide erfasst. Für jede Person im Sample liegt eine Einstufung der Big-Five-Merkmale zwischen 1 (= unzutreffend) und 5 (= zutreffend) vor.

Der Standort eines Probanden wird im Rahmen der Internet-Befragung über die Postleitzahl erhoben (,Was ist die Postleitzahl des Ortes, in dem Sie derzeit leben?"). Daneben wird auch der Wohnort während der Jugendzeit eines Probanden erfasst (,Wie lautet die Postleitzahl des Ortes, an dem Sie Ihre Jugend überwiegend verbrachten?"), sodass sich hieraus Rückschlüsse auf das Mobilitätsverhalten ziehen lassen. ${ }^{4}$ Unter der Annahme, dass die Persönlichkeitsmerkmale im Zeitverlauf weitgehend konstant sind, lassen sich auf dieser Grundlage auch Aussagen zum Beitrag der Mobilität zur Persönlichkeitsstruktur der Bevölkerung einer Region treffen.

Für den Gesamtindikator der unternehmerischen Persönlichkeitsstruktur werden die Einstufungen zu den einzelnen Big-Five-Merkmalen zunächst in eine Skala von 0 bis 4

\footnotetext{
3 http://www.outofservice.com (18.10.2017), vgl. auch Bleidorn/ Klimstra/Denissen et al. (2013), zur deutschsprachigen Version der Homepage vgl. http://de.outofservice.com/bigfive (18.10.2017).

${ }_{4}$ Weiterhin wird gefragt, wie lange und wie gerne man an dem betreffenden Standort gelebt hat.
} 
transformiert. ${ }^{5}$ Obschonka, Schmitt-Rodermund, Silbereisen et al. (2013) folgend wird dann die individuelle unternehmerische Persönlichkeitsstruktur über ein Abweichungsmaß zu einem fixen Referenzprofil berechnet. Dieses Referenzprofil hat den höchstmöglichen Wert für Offenheit für Erfahrungen, Extraversion und Gewissenhaftigkeit (4) sowie den kleinstmöglichen Wert für Neurotizismus und Verträglichkeit (0). Für den Gesamtindikator der individuellen unternehmerischen Persönlichkeitsstruktur werden dann alle Abweichungen der Big Five einer Person von diesem Referenzprofil quadriert (um negative Werte auszuschließen) und aufaddiert. Ein negatives Vorzeichen für diesen Gesamtindikator soll verdeutlichen, dass es sich um eine Abweichung von einem idealen Referenzwert handelt. Je näher dieser Wert bei 0 liegt, desto eher ist das Persönlichkeitsprofil als ,unternehmerisch ${ }^{\text { anzusehen. }}{ }^{6}$

\subsection{Stichprobe und Repräsentativität}

Wir beschränken unseren Vergleich der Persönlichkeitsstruktur auf die erwerbsfähige Bevölkerung im Alter von 20 bis 64 Jahren im Zeitraum 2003-2015. Für insgesamt 67.328 Personen dieser Gruppe liegen uns Informationen zur Postleitzahl des aktuellen Wohnorts sowie zum Wohnort während der Jugendzeit vor. Den räumlichen Bezugsrahmen stellen die deutschen Raumordnungsregionen in der Abgrenzung und Klassifikation des Bundesinstituts für Bau-, Stadt- und Raumforschung dar. ${ }^{7}$ Eine Raumordnungsregion ist eine funktionale Raumeinheit, die in der Regel mindestens eine kreisfreie Stadt sowie mehrere umliegende Kreise umfasst. Insgesamt ist Deutschland in 96 Raumordnungsregionen gegliedert. Da die Stadtstaaten Hamburg und Bremen zwar als separate Raumordnungsregionen abgegrenzt sind, aber keine funktionalen Raumeinheiten darstellen, haben wir sie mit umliegenden Kreisen zusammengefasst. ${ }^{8}$ Durch diese Zusammenfassung reduziert sich die Anzahl der Regionen in unserer Analyse auf 93. Diese Regionen sind in siedlungsstrukturelle Regi-

\footnotetext{
${ }^{5}$ Das heißt, von den Originalwerten (1 bis 5) wird jeweils der Wert 1 subtrahiert.

${ }^{6}$ Die Formel zur Berechnung der unternehmerischen Persönlichkeit ausgehend von den Originalwerten der Befragung lautet: Unternehmerisches Persönlichkeitsprofil $=-1 *(4-($ Offenheit- $)) *(4-($ Offenheit$1))+(4-($ Extraversion- $)) *(4-($ Extraversion- 1$))+(4-($ Gewissenhaftigkeit-1))*(4-(Gewissenhaftigkeit-1)) + (Verträglichkeit-1) * (Verträglichkeit-1) + (Neurotizismus-1) * (Neurotizismus-1)).

7 http://www.bbsr.bund.de/BBSR/DE/Raumbeobachtung/ Raumabgrenzungen/SiedlungsstrukturelleGebietstypen/Regionstypen/ regionstypen.html (18.10.2017).

${ }^{8}$ Die Raumordnungsregion Hamburg wurde mit den Raumordnungsregionen Schleswig-Holstein Süd und Hamburg-Umland-Süd zusammengefasst. Bremen und Bremen-Umland bilden eine Region.
}

onstypen - Agglomerationsräume, Regionen mit Verdichtungsansätzen und Ländliche Regionen - untergliedert. ${ }^{9}$

In der Analyse berücksichtigen wir nur Personen im Alter zwischen 20 und 64 Jahren. Wahrscheinlich bedingt durch die Datenerhebung via Internet sind jüngere Teilnehmer an der Studie überrepräsentiert. Das Durchschnittsalter der Probanden beträgt 29,75 Jahre (Standardabweichung = 12,01). Rund $59 \%$ der Teilnehmer waren weiblich (Standardabweichung $=0,49$ ). Dies entspricht in etwa den im Rahmen des Projektes für die USA erhobenen Daten (Rentfrow/Gosling/Potter 2008). ${ }^{10}$ In der empirischen Analyse kann die Verzerrung, die sich eventuell aus der Überrepräsentation bestimmter Altersgruppen ergibt, durch eine Gewichtung der einzelnen Beobachtungen auf Basis der Altersstruktur korrigiert werden. Dabei werden den Beobachtungen Gewichte zugewiesen, die der inversen Wahrscheinlichkeit entsprechen, in der Stichprobe enthalten zu sein (Horvitz/Thompson 1952; Elliott 2008). In unserem Falle wird diese Wahrscheinlichkeit durch den Bevölkerungsanteil der Altersgruppe repräsentiert, der ein Teilnehmer angehört. Dabei nehmen wir eine zusätzliche Unterscheidung nach dem Geschlecht vor. Die Daten zur Bevölkerungsstruktur nach Geschlecht und Altersgruppen (fünf Kategorien) stammen aus dem Online-Angebot des Statistischen Bundesamts und beziehen sich auf das Jahr 2010. ${ }^{11}$ So wird beispielsweise Frauen im Alter zwischen 20 und 24 Jahren als Gewicht der Wert zugeordnet, der sich aus der Division von 1 durch den regionalen Anteil der Frauen zwischen 20 und 24 Jahren an der weiblichen Gesamtbevölkerung einer Region ergibt.

Alternativ nutzen wir ein Gewichtungsverfahren, das an der Abweichung des Anteils einer Altersgruppe im Sample vom Bevölkerungsanteil in der jeweiligen Gesamtbevölkerung einer Region ansetzt. Fällt etwa der Anteil der 20- bis 24-jährigen Teilnehmer in der Stichprobe doppelt so hoch aus wie in der Bevölkerung insgesamt, so wird dieser Gruppe der Wert 0,5 zugewiesen. Ist umgekehrt der Anteil der 55- bis 59-Jährigen in der Auswahl nur halb so hoch wie in der Gesamtbevölkerung, so wird ein Gewicht von 2 ver-

\footnotetext{
${ }_{9}$ Insgesamt 36.126 Personen aus unserem Sample sind in Agglomerationsräumen angesiedelt. Aus Regionen mit Verdichtungsansätzen liegen 19.938 Beobachtungen vor. 11.264 der Personen unseres Stichprobe leben in ländlichen Regionen.

10 Zur Repräsentativität der in Deutschland erhobenen Daten vgl. Obschonka/Stuetzer/Rentfrow et al. (2017). Darüber hinaus muss die Selbstselektivität der Teilnahme an der Befragung berücksichtigt werden.

${ }^{11}$ Das Bezugsjahr 2010 wurde deshalb gewählt, weil es in etwa in der Mitte des Zeitraums 2003-2015 liegt, für den uns Antworten zu den Persönlichkeitsmerkmalen vorliegen. Etwa $47 \%$ aller Antworten stammen aus dem Zeitraum 2003-2009; entsprechend haben zirka $53 \%$ aller Teilnehmer zwischen 2010 und 2015 an der Befragung teilgenommen.
} 
geben. Wir berichten nur die Ergebnisse nach dem zweiten (alternativen) Verfahren. ${ }^{12}$

Um zu zeigen, dass alle Raumordnungsregionen angemessen im Sample vertreten sind, korrelieren wir den Bevölkerungsanteil einzelner Regionen an der Gesamtbevölkerung Deutschlands mit den jeweiligen regionalen Anteilen der Stichprobe an der gesamten Auswahl-Population. Hierbei ergibt sich ein annähernd proportionaler Zusammenhang $\left(r=0,96^{13}\right)$, der in etwa den Ergebnissen von Rentfrow/Gosling/Potter (2008) für die USA entspricht. Auch der Anteil der weiblichen Probanden in den einzelnen Regionen entspricht recht gut den entsprechenden Anteilen an der jeweiligen regionalen Bevölkerung $\left(r=0,95^{14}\right)$. Schließlich vergleichen wir auch die Qualifikationsstruktur der Sample-Population anhand von Angaben aus der Beschäftigtenstatistik des Instituts für Arbeitsmarkt- und Berufsforschung (Betriebs-Historik-Panel; Gruhl/Schmucker/ Seth 2012) mit der jeweiligen regionalen Grundgesamtheit aller sozialversicherungspflichtig Beschäftigten. Es zeigt sich eine Korrelation zwischen den Personen mit tertiärem Bildungsabschluss und dem entsprechenden regionalen Beschäftigungsanteil von $r=0,68^{15}$. Unsere Analysen zur Repräsentativität weisen darauf hin, dass unsere Stichprobe für Deutschland qualitativ vergleichbar mit den Daten für die USA ist, die in der Vergangenheit bereits erfolgreich in der psychologischen Forschung genutzt wurden (z. B. Rentfrow/Gosling/Potter 2008). Bei unserer Betrachtung stützen wir uns im Wesentlichen auf Mittelwertvergleiche der gewichteten Ausprägung der Persönlichkeitsmerkmale der Bewohner verschiedener Regionen.

\section{Räumliche Unterschiede in der Verteilung von unternehmerischen Persönlichkeiten}

\subsection{Die psychologische Landkarte Deutschlands}

Abbildung 1 zeigt die durchschnittliche Abweichung einer unternehmerischen Persönlichkeit vom Referenzprofil der Bevölkerung in den deutschen Raumordnungsregionen. Tabelle 1 enthält den genauen Wert pro Raumordnungsregion. Aus der Konstruktion des Indikators als Abweichung vom statistischen Referenztyp (vgl. Kapitel 2.1) ergibt sich, dass der entsprechende Wert jeweils ein negatives Vorzeichen aufweist. Je geringer die negative Ausprägung des entsprechenden Wertes, desto stärker ausgebildet ist das unternehmerische Persönlichkeitsprofil. Dabei bleibt fest-

\footnotetext{
12 Die Ergebnisse nach dem ersten Verfahren unterscheiden sich kaum von den hier präsentierten Resultaten.

13 Statistisch signifikant auf dem $1 \%$-Niveau.

${ }^{14}$ Statistisch signifikant auf dem $1 \%$-Niveau.

${ }^{15}$ Statistisch signifikant auf dem $1 \%$-Niveau.
}

Tabelle 1 Ausprägung des unternehmerischen Persönlichkeitsprofils (durchschnittliche Abweichung vom Referenzprofil einer unternehmerischen Persönlichkeit) in den 96 Untersuchungsregionen im Zeitraum 2003-2015

\begin{tabular}{|c|c|c|}
\hline \multicolumn{2}{|c|}{ Raumordnungsregion } & \multirow{2}{*}{$\begin{array}{l}\begin{array}{l}\text { Profil unternehmeri- } \\
\text { scher Persönlichkeit }\end{array} \\
-19.568\end{array}$} \\
\hline 101 & Schleswig-Holstein Mitte & \\
\hline 102 & Schleswig-Holstein Nord & -19.770 \\
\hline 103 & Schleswig-Holstein Ost & -19.624 \\
\hline 104 & Schleswig-Holstein Süd* & -18.393 \\
\hline 105 & Schleswig-Holstein Süd-West & -21.215 \\
\hline 201 & Hamburg* & -18.393 \\
\hline 301 & Braunschweig & -19.629 \\
\hline 302 & Bremen-Umland* & -19.328 \\
\hline 303 & Bremerhaven & -21.215 \\
\hline 304 & Emsland & -20.079 \\
\hline 305 & Göttingen & -19.484 \\
\hline 306 & Hamburg-Umland-Süd* & -18.393 \\
\hline 307 & Hannover & -18.893 \\
\hline 308 & Hildesheim & -19.339 \\
\hline 309 & Lüneburg & -19.123 \\
\hline 310 & Oldenburg & -19.163 \\
\hline 311 & Osnabrück & -19.465 \\
\hline 312 & Ostfriesland & -19.293 \\
\hline 313 & Südheide & -20.667 \\
\hline 401 & Bremen* & -19.328 \\
\hline 501 & Aachen & -19.306 \\
\hline 502 & Arnsberg & -19.417 \\
\hline 503 & Bielefeld & -19.420 \\
\hline 504 & Bochum/Hagen & -19.399 \\
\hline 505 & Bonn & -19.043 \\
\hline 506 & Dortmund & -19.528 \\
\hline 507 & Duisburg/Essen & -19.624 \\
\hline 508 & Düsseldorf & -18.657 \\
\hline 509 & Emscher-Lippe & -19.621 \\
\hline 510 & Köln & -18.747 \\
\hline 511 & Münster & -19.919 \\
\hline 512 & Paderborn & -19.385 \\
\hline 513 & Siegen & -19.128 \\
\hline 601 & Mittelhessen & -19.847 \\
\hline 602 & Nordhessen & -19.209 \\
\hline
\end{tabular}

zuhalten, dass es sich hierbei um ein stereotypisches Profil handelt. Sicherlich gibt es erfolgreiche Unternehmer, die ein deutlich anderes Big-Five-Profil aufweisen, wie es auch viele Personen mit einem unternehmerischen Profil gibt, die nicht unternehmerisch tätig werden. Dennoch stellt dieses Profil ein zwar relativ allgemeines, aber gut validiertes und erforschtes $\mathrm{Ma} ß$ der allgemeinen unternehmerischen Persönlichkeit dar (für eine weiterführende Diskussion vgl. Obschonka/Schmitt-Rodermund/Silbereisen et al. 2013).

Aus der Abbildung wird unmittelbar ersichtlich, dass es systematische regionale Unterschiede in der Ausprägung ei- 
Tabelle 1 Ausprägung des unternehmerischen Persönlichkeitsprofils (durchschnittliche Abweichung vom Referenzprofil einer unternehmerischen Persönlichkeit) in den 96 Untersuchungsregionen im Zeitraum 2003-2015 (Fortsetzung)

\begin{tabular}{|c|c|c|}
\hline \multicolumn{2}{|c|}{ Raumordnungsregion } & \multirow{2}{*}{$\begin{array}{l}\text { Profil unternehmeri- } \\
\text { scher Persönlichkeit } \\
-18.951\end{array}$} \\
\hline 603 & Osthessen & \\
\hline 604 & Rhein-Main & -18.621 \\
\hline 605 & Starkenburg & -19.020 \\
\hline 701 & Mittelrhein-Westerwald & -19.543 \\
\hline 702 & Rheinhessen-Nahe & -19.237 \\
\hline 703 & Rheinpfalz & -18.974 \\
\hline 704 & Trier & -19.388 \\
\hline 705 & Westpfalz & -18.616 \\
\hline 801 & Bodensee-Oberschwaben & -18.714 \\
\hline 802 & Donau-Iller (Baden-Württemberg) & -19.406 \\
\hline 803 & Franken & -18.630 \\
\hline 804 & Hochrhein-Bodensee & -18.868 \\
\hline 805 & Mittlerer Oberrhein & -18.962 \\
\hline 806 & Neckar-Alb & -19.703 \\
\hline 807 & Nordschwarzwald & -19.124 \\
\hline 808 & Ostwürttemberg & -18.600 \\
\hline 809 & Schwarzwald-Baar-Heuberg & -19.317 \\
\hline 810 & Stuttgart & -18.580 \\
\hline 811 & Südlicher Oberrhein & -18.972 \\
\hline 812 & Unterer Neckar & -19.199 \\
\hline 901 & Allgäu & -19.483 \\
\hline 902 & Augsburg & -19.134 \\
\hline 903 & Bayerischer Untermain & -18.220 \\
\hline 904 & Donau-Iller (Bayern) & -19.367 \\
\hline 905 & Donau-Wald & -19.734 \\
\hline 906 & Industrieregion Mittelfranken & -19.164 \\
\hline 907 & Ingolstadt & -19.354 \\
\hline 908 & Landshut & -19.383 \\
\hline 909 & Main-Rhön & -19.102 \\
\hline 910 & München & -18.381 \\
\hline 911 & Oberfranken-Ost & -20.175 \\
\hline 912 & Oberfranken-West & -19.672 \\
\hline 913 & Oberland & -18.691 \\
\hline 914 & Oberpfalz-Nord & -20.500 \\
\hline 915 & Regensburg & -19.083 \\
\hline
\end{tabular}

ner unternehmerischen Persönlichkeitsstruktur in Deutschland gibt. Besonders ins Auge fallen die relativ hohen Werte in den Regionen von Baden-Württemberg, die dem weit verbreiteten Klischee vom unternehmerischen Schwaben und Badener gut zu entsprechen scheinen. Weitere Regionen, in denen die Durchschnittswerte für die unternehmerische Persönlichkeitsstruktur relativ hoch sind, sind etwa München, Oberland, Bayerischer Untermain, Rhein-Main, Rheinhessen-Nahe, Köln, Düsseldorf, Hannover und Hamburg. Regionen, in denen das unternehmerische Persönlichkeitsprofil in der Gesamtbevölkerung relativ gering ausge-
Tabelle 1 Ausprägung des unternehmerischen Persönlichkeitsprofils (durchschnittliche Abweichung vom Referenzprofil einer unternehmerischen Persönlichkeit) in den 96 Untersuchungsregionen im Zeitraum 2003-2015 (Fortsetzung)

\begin{tabular}{|c|c|c|}
\hline \multicolumn{2}{|c|}{ Raumordnungsregion } & \multirow{2}{*}{$\begin{array}{l}\text { Profil unternehmeri- } \\
\text { scher Persönlichkeit } \\
-19.335\end{array}$} \\
\hline 916 & Südostoberbayern & \\
\hline 917 & Westmittelfranken & -19.065 \\
\hline 918 & Würzburg & -19.523 \\
\hline 1001 & Saar & -19.611 \\
\hline 1101 & Berlin & -19.161 \\
\hline 1201 & Havelland-Fläming & -18.961 \\
\hline 1202 & Lausitz-Spreewald & -19.594 \\
\hline 1203 & Oderland-Spree & -19.468 \\
\hline 1204 & Prignitz-Oberhavel & -19.250 \\
\hline 1205 & Uckermark-Barnim & -20.934 \\
\hline 1301 & Mecklenburgische Seenplatte & -20.160 \\
\hline 1302 & Mittleres Mecklenburg/Rostock & -19.413 \\
\hline 1303 & Vorpommern & -19.722 \\
\hline 1304 & Westmecklenburg & -19.317 \\
\hline 1401 & Oberes Elbtal/Osterzgebirge & -19.409 \\
\hline 1402 & Oberlausitz-Niederschlesien & -19.468 \\
\hline 1403 & Südsachsen & -19.231 \\
\hline 1404 & Westsachsen & -19.707 \\
\hline 1501 & Altmark & -19.781 \\
\hline 1502 & Anhalt-Bitterfeld-Wittenberg & -20.553 \\
\hline 1503 & Halle/Saale & -19.852 \\
\hline 1504 & Magdeburg & -19.103 \\
\hline 1601 & Mittelthüringen & -19.637 \\
\hline 1602 & Nordthüringen & -19.538 \\
\hline 1603 & Ostthüringen & -19.400 \\
\hline 1604 & Südthüringen & -20.354 \\
\hline
\end{tabular}

Werte beziehen sich auf die im Datensatz vorgenommenen Anpassungen.

*Die Raumordnungsregionen 104, 201, 306 sowie die Regionen 302 und 401 wurden jeweils zu einer Region zusammengefasst.

prägt ist, umfassen unter anderem solche Gebiete, die lange Zeit von großen Unternehmen der Kohle- und Stahlindustrie dominiert waren, wie das Ruhrgebiet und das Saarland. Auffällig ist auch die geringe Ausprägung des unternehmerischen Persönlichkeitsprofils im nördlichen SchleswigHolstein, im südlichen Teil Sachsen-Anhalts sowie im Osten von Franken und Bayern.

Die relativ schwache Ausprägung unternehmerischer Persönlichkeitsstrukturen in der Bevölkerung in Regionen, in denen Großunternehmen eine dominierende Rolle innehaben bzw. -hatten, wird in der Literatur vor allem auf die geringe Anzahl oder Dichte von unternehmerischen Rollenmodellen und entsprechenden Peer-Effekten in der Region zurückgeführt (Chinitz 1961). Empirische Untersuchungen für Großbritannien (Stuetzer/Obschonka/Audretsch et al. 2016) und die USA (Glaeser/Kerr/Kerr 2015) stützen diese Vermutung. Demgegenüber erweist sich die Prä- 
Abbildung 1 Regional unterschiedliche Verteilung der Erwerbsbevölkerung mit unternehmerischem Persönlichkeitsprofil

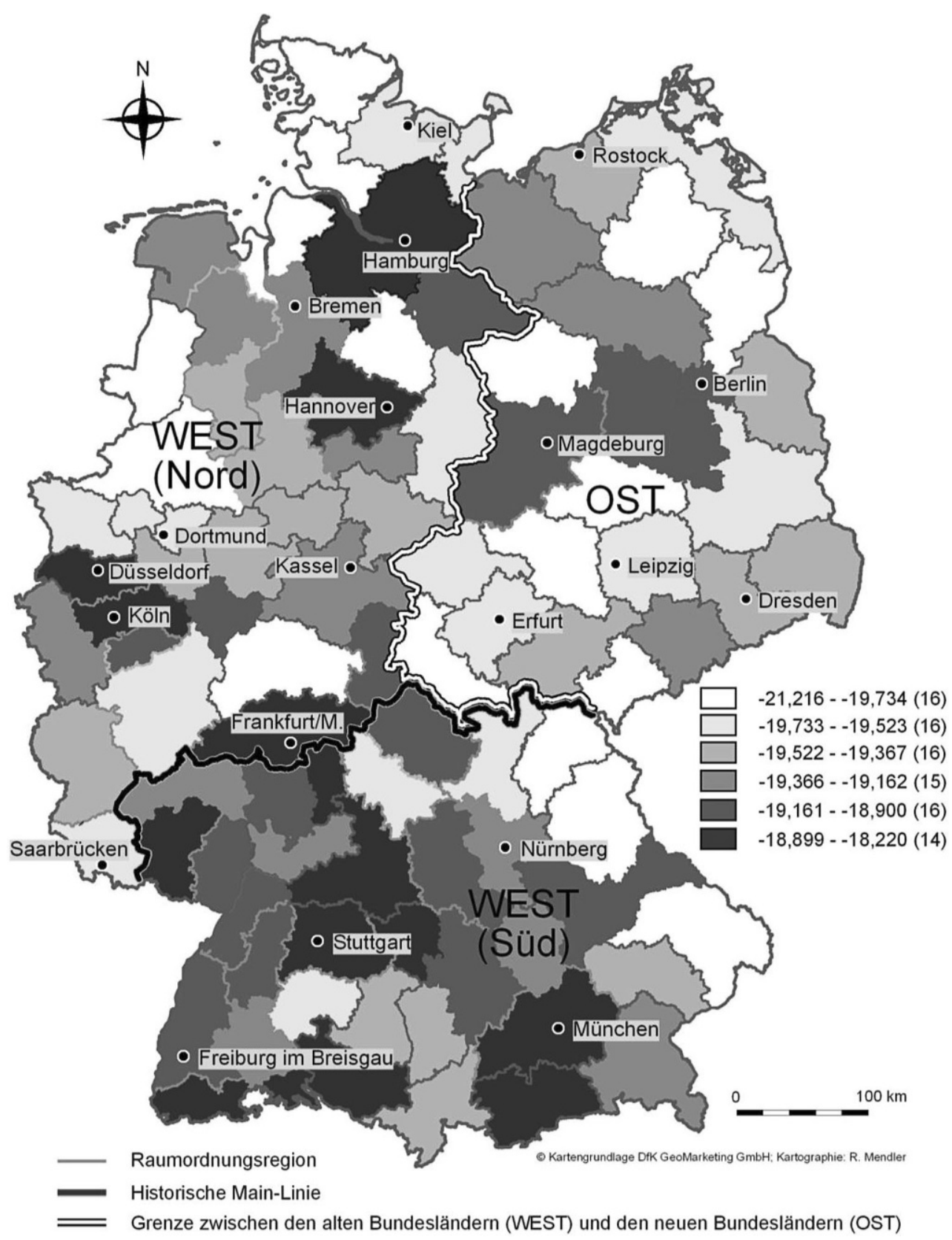

senz von vielen Kleinunternehmen aufgrund der regionalen Häufigkeit von unternehmerischen Rollenmodellen als förderlich (Parker 2009; Bosma/Hessels/Schutjens et al. 2012; Sorenson 2017), was auch als „Saatbeet-Funktion“ von Kleinunternehmen charakterisiert wird (Beesley/Hamilton 1984).

Für einen Vergleich der regionalen Ausprägung einer unternehmerischen Persönlichkeitsstruktur mit dem Niveau der regionalen Gründungsaktivitäten berechnen wir regionale Gründungsraten (Anzahl der Gründungen pro 10.000 Personen in der erwerbsfähigen Bevölkerung). Die Informationen über die Gründungen beruhen auf dem Mann- heimer Unternehmenspanel ${ }^{16}$ für die Jahre 2000 bis 2013. Abbildung 2 zeigt die Mittelwerte für die regionale Gründungsrate in den deutschen Raumordnungsregionen. Regionen mit relativ hohen Gründungsraten befinden sich vor al-

\footnotetext{
16 Das Mannheimer Unternehmenspanel wird vom Zentrum für Europäische Wirtschaftsforschung (ZEW) aufbereitet und umfasst sämtliche Unternehmensgründungen, die über die Rating-Agentur Creditreform erfasst sind (vgl. Bersch/Gottschalk/Müller et al. 2014). Die relativ geringen Werte für die ostdeutschen Regionen sind wahrscheinlich zum Teil dadurch bedingt, dass diese Datenquelle keine Gründungen von Zweigbetrieben enthält und sehr kleine Gründungen unterrepräsentiert sind. $\mathrm{Zu}$ einer Analyse der Gründungsaktivität in Ostdeutschland vgl. Fritsch/Bublitz/Sorgner et al. (2014).
} 
Abbildung 2 Durchschnittliche regionale Gründungsraten in den deutschen Raumordnungsregionen im Zeitraum 20002013

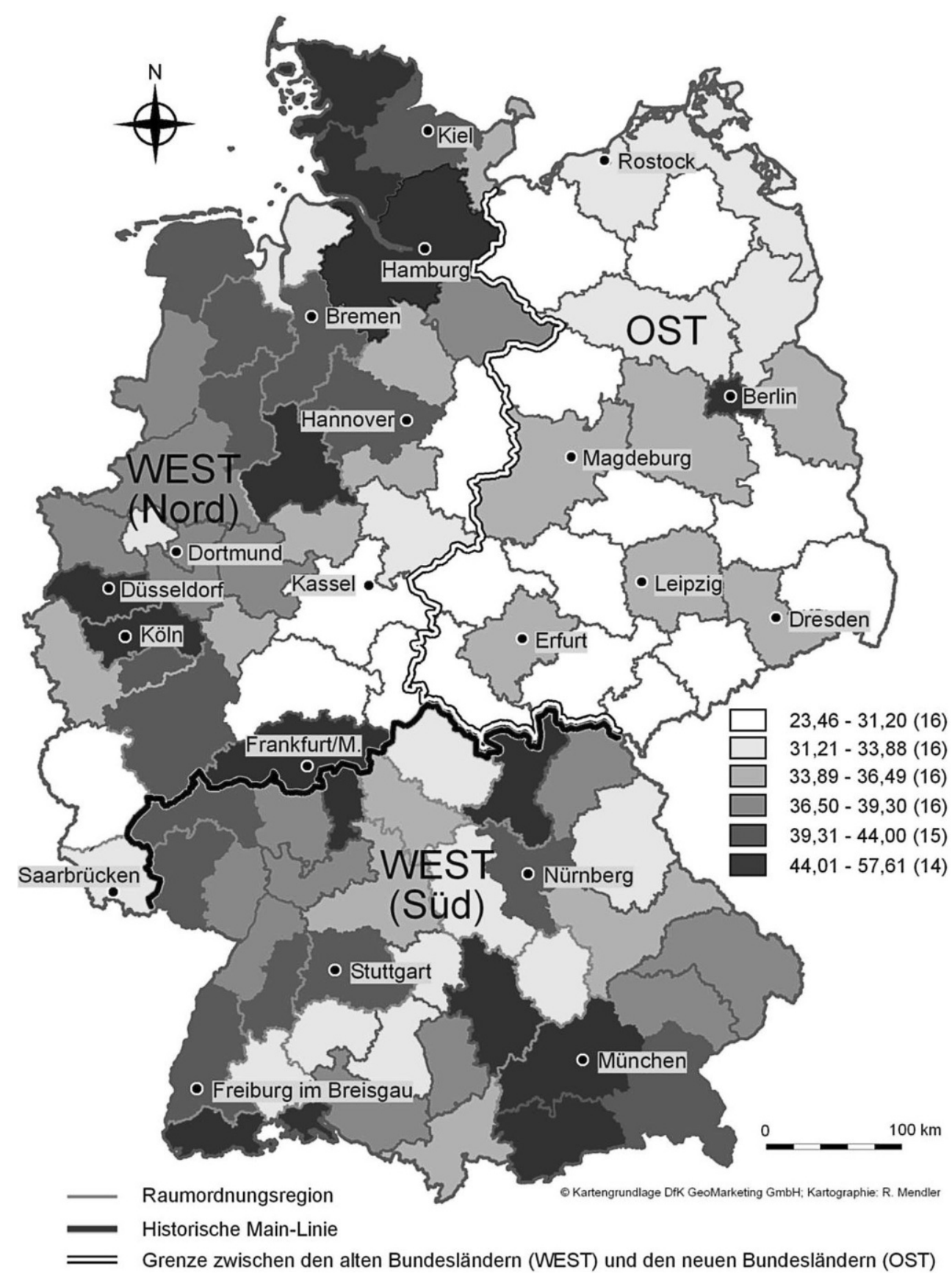

lem in den alten Bundesländern, wobei insbesondere großstädtisch geprägte Regionen wie München, Frankfurt am Main, Köln, Düsseldorf und Hamburg ein hohes Niveau an Gründungsaktivität aufweisen. In Ostdeutschland sind lediglich für Berlin ähnlich hohe Werte zu verzeichnen. Die Korrelation zwischen dem durchschnittlichen regionalen unternehmerischen Persönlichkeitsprofil und der regionalen Gründungsrate beträgt $r=0,42^{17}$. Dieser für eine Querschnittsbetrachtung relativ hohe Wert zeigt, dass ein deutlicher Zusammenhang zwischen der ,regionalen Grün-

\footnotetext{
17 Statistisch signifikant auf dem 1\%-Niveau.
}

dungsneigung' und den als unternehmerisch klassifizierten Persönlichkeitsmerkmalen der regionalen Bevölkerung besteht. Diese deutliche Korrelation spiegelt sich auch in der Ähnlichkeit mit dem in Abbildung 1 dargestellten räumlichen Muster des durchschnittlichen unternehmerischen Persönlichkeitsprofils wider.

\subsection{Unterschiede nach Verdichtungsgrad}

Vor allem in der angelsächsischen Literatur ist die Vorstellung verbreitet, dass große Städte gute Voraussetzungen für Unternehmertum bieten und daher eine hohe Anziehungs- 


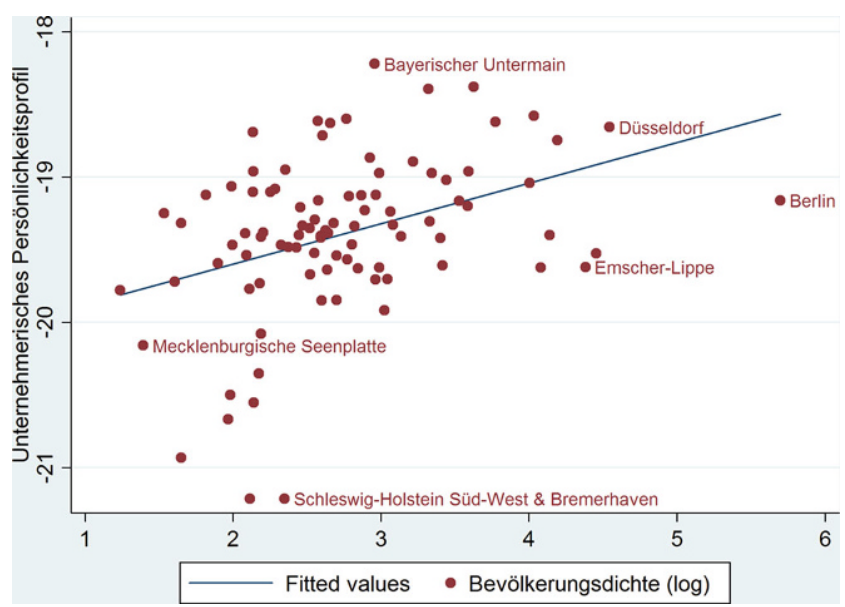

Abbildung 3 Bevölkerungsdichte (Einwohner/Fläche in $\mathrm{km}^{2}$ ) und unternehmerische Persönlichkeitsstrukturen in den deutschen Raumordnungsregionen

kraft auf Personen mit unternehmerischen Persönlichkeitsmerkmalen ausüben (Florida 2002; Glaeser 2011a; Glaeser 2011b; Bosma/Sternberg 2014; Florida/Adler/Mellander 2017). Gute Voraussetzungen für unternehmerische Selbstständigkeit - so die Argumentation - ergäben sich vor allem aus dem für Städte kennzeichnenden hohen $\mathrm{Ma}$ an vorhandenem Wissen und der Intensität von Wissens-Spillovern. Weitere wesentliche Vorteile für Gründungen in Städten seien die gute Verfügbarkeit von wichtigen Ressourcen wie etwa Arbeitskräfte mit speziellen Qualifikationen (Helsley/ Strange 2011) sowie von Finanzierungsangeboten. Weiterhin wird vermutet, dass große Städte durch ein vergleichsweise hohes $\mathrm{Ma} ß$ an Extraversion und Offenheit der Bevölkerung für neue Erfahrungen gekennzeichnet sind (Florida 2002).

Abbildung 3 zeigt den Zusammenhang zwischen der regionalen Bevölkerungsdichte und der Ausprägung unternehmerischer Persönlichkeitsstrukturen. Tabelle 2 gibt einen entsprechenden Überblick für städtische Regionen, ländliche Regionen und Regionen mit Verdichtungsansätzen. Im Einklang mit der vielfach in der Literatur zu findenden Vermutung (Glaeser 2011a; Glaeser 2011b; Florida/Adler/Mellander 2017) weist die Bevölkerung in städtischen Regionen tatsächlich eine deutlich stärker ausgeprägte unternehmerische Persönlichkeitsstruktur auf als die Bevölkerung in ländlich geprägten Regionen und in Regionen mit Verdichtungsansätzen. Die Unterschiede zwischen den städtischen Regionen einerseits und den ländlich geprägten Regionen sowie den Regionen mit Verdichtungsansätzen andererseits sind statistisch hochsignifikant. Demgegenüber lässt sich zwischen den Regionen mit Verdichtungsansätzen und den ländlichen Regionen kein statistisch signifikanter Unterschied feststellen (vgl. Tabelle 2).
Da die statistische Signifikanz eines Unterschiedes zwischen zwei Stichproben (abhängig von der Anzahl an Beobachtungen) nicht auf das Ausmaß des Unterschiedes schließen lässt, ziehen wir als Maß für die Effektstärke Cohens d (vgl. Cohen 1988; Bortz/Schuster 2010: 108) heran. Je höher der Wert dieser Maßzahl ist, desto größer ist der Unterschied zwischen den jeweils betrachteten Regionstypen. Nach Cohen (1988) zeigt ein Wert von d zwischen 0,2 und 0,5 einen gering ausgeprägten Effekt an, während der Effekt bei einem d-Wert zwischen 0,5 und 0,8 als von mittlerer Stärke und bei einem Wert größer als 0,8 als stark einzustufen ist. Entsprechend dieser Klassifikation zeigen die hier ermittelten d-Werte (vgl. Tabelle 3) einen relativ schwachen Effekt des Regionstyps an. Als weiteres Maß für die Effektstärke berechnen wir den Anteil der Mittelwertunterschiede an der gemeinsamen Standardabweichung. ${ }^{18}$ Wie sich zeigt, erklärt der Mittelwertunterschied hinsichtlich unternehmerischer Persönlichkeitsprofile zwischen städtischen Regionen und Regionen mit Verdichtungsansätzen etwa 3,6\% (Zeile (a) - (b)) der gesamten Streuung des unternehmerischen Persönlichkeitsprofils der Personen aus beiden Regionstypen. Vergleicht man städtische und ländliche Regionen (Zeile (a) - (c)), so beträgt dieser Anteil etwa 3,8\%. Die Mittelwertunterschiede zwischen Regionen mit Verdichtungsansätzen und ländlichen Regionen (Zeile (b) (c)) wiederum entsprechen lediglich knapp einem Viertelprozent der gemeinsamen Streuung des unternehmerischen Persönlichkeitsprofils der Personen in beiden Regionstypen. Insgesamt lässt sich feststellen, dass die Stadt-LandUnterschiede als recht gering einzuschätzen sind. Dieser geringe Unterschied könnte unter anderem dadurch bedingt sein, dass die in den beiden Regionstypen zusammengefassten Räume hinsichtlich der Ausprägung unternehmerischer Persönlichkeitsstrukturen sehr heterogen sind (vgl. Abbildung 1), was dazu führt, dass ein wesentlicher Teil dieser Unterschiede bei der Betrachtung von Regionstypen verdeckt bleibt.

Der Zusammenhang zwischen Bevölkerungsdichte und der Ausprägung unternehmerischer Persönlichkeitsprofile (vgl. Abbildung 3) lässt sich anhand von OLSRegressionsmodellen ${ }^{19}$ veranschaulichen. Modell I in Tabelle 4 zeigt einen statistisch signifikanten Zusammenhang zwischen der regionalen Ausprägung eines unternehmerischen Persönlichkeitsprofils und der regionalen Gründungsrate. Führt man die Bevölkerungsdichte in das Modell ein (Modell II in Tabelle 4), so reduziert sich der Koeffizient für das unternehmerische Profil etwas, bleibt aber statistisch

\footnotetext{
${ }^{18}$ Bei der Berechnung von Cohens d wird die Mittelwertabweichung durch die halbierte gemeinsame Standardabweichung geteilt und um einen kleinen, positiven Bias korrigiert. Die dargestellten Maße sind somit relativ ähnlich.

19 Ordinary-Least-Squares-Modell; Schätzmodell.
} 
Tabelle 2 Stadt-Land-Unterschiede bezüglich unternehmerischer Persönlichkeitsstrukturen (gewichtete Ergebnisse)

\begin{tabular}{llll}
\hline & (a) Städtische Regionen & (b) Regionen mit Verdichtungsansätzen & $\begin{array}{l}\text { (c) Ländliche Regionen } \\
\text { (N=11.577) }\end{array}$ \\
Mittelwert & $(\mathrm{N}=42.029)$ & $(\mathrm{N}=20.150)$ & $-18,759$ \\
Standardabweichung & $-18,496$ & $-18,744$ & 6,935 \\
(a) Städtische Regionen & 6,854 & 6,937 & $* *(* * *)$ \\
(b) Regionen mit Verdichtungsansätzen & $* *(* *)$ & $* *(* *)$ & n.s. (n.s.) \\
(c) Ländliche Regionen & $* *(* * *)$ & - & - \\
\hline
\end{tabular}

Im unteren Teil der Tabelle ist angegeben, inwiefern die Unterschiede der Mittelwerte beim Vorkommen unternehmerischer Persönlichkeitsprofile zwischen den Regionstypen statistisch signifikant sind. Die statistische Signifikanz von Unterschieden der entsprechenden Standardabweichungen ist jeweils in Klammern angegeben. Der Test auf statistische Signifikanz für die Unterschiede der Standardabweichungen kann nur auf der Basis ungewichteter Werte durchgeführt werden. ***: statistisch signifikant auf dem 0,1\%-Niveau; **: statistisch signifikant auf dem $1 \%$-Niveau; *: statistisch signifikant auf dem 5\%-Niveau; n.s.: Unterschied nicht statistisch signifikant.

Tabelle 3 Anteil der Stadt-Land-Unterschiede an den gesamten Unterschieden bezüglich der unternehmerischen Persönlichkeitsstruktur (gewichtete Ergebnisse)

\begin{tabular}{lll}
\hline & Cohens d & $\begin{array}{l}\text { Anteil der Mittelwertunteschiede an der } \\
\text { gemeinsamen Standardabweichung (\%) }\end{array}$ \\
(a) - (b) & 0,036 & 3,605 \\
(a) - (c) & 0,038 & 3,834 \\
(b) - (c) & 0,002 & 0,221 \\
\hline
\end{tabular}

signifikant. Der Zusammenhang zwischen Bevölkerungsdichte und regionaler Gründungsrate ist signifikant positiv und er wird stärker, wenn man nicht auf regionale Unterschiede bezüglich des Vorkommens unternehmerischer Persönlichkeiten kontrolliert (Modell III). Die Modelle enthalten Dummy-Variablen für die einzelnen Bundesländer, um auf Unterschiede bezüglich der Förderung von Gründungen zu kontrollieren. ${ }^{20}$

Neben Angaben zum aktuellen Wohnort (Postleitzahl) enthalten die Daten auch die Information, wo ein Proband während seiner Jugend gelebt hat. Dies eröffnet die Möglichkeit, den Einfluss von räumlicher Mobilität näher zu untersuchen. Aus Gründen der Übersichtlichkeit beschränken wir uns bei dieser Analyse auf die Gegenüberstellung der beiden polaren Raumtypen „Stadt“ (hochverdichtete städtische Raumordnungsregionen; Agglomerationsräume) und „Land“ (ländliche Raumordnungsregionen). Abbildung 4 gibt einen Überblick über die Verteilung unternehmerischer Persönlichkeitsstrukturen in „Stadt"- und „Land“-Regionen sowie zu den Raumabgrenzungen, die in den Kapiteln 3.3 und 3.4 betrachtet werden.

In den beiden linken Spalten von Tabelle 5 werden Persönlichkeitsprofile von Probanden nach ihrem Wohnort in „Stadt“ oder „Land“ während ihrer Jugend (Spalten a und b) sowie zum Zeitpunkt der Befragung (Spalten (c) und (d)) miteinander verglichen. Dabei zeigt sich, dass ein statistisch signifikanter Stadt-Land-Unterschied nur hinsicht-

${ }^{20}$ Die Nichtberücksichtigung von Bundesland-Dummies führt zu keinen grundsätzlich anderen Ergebnissen.
Tabelle 4 Regressionsanalyse zum Zusammenhang zwischen Gründungsraten, Ausprägung des unternehmerischen Persönlichkeitsprofils und Bevölkerungsdichte (OLS)

\begin{tabular}{llll}
\hline & I & II & III \\
Unternehmerisches & $4,352 * * *$ & $3,128 * * *$ & - \\
Persönlichkeitsprofil & $(1,187)$ & $(1,095)$ & \\
Bevölkerungsdichte & - & $3,758 * * *$ & $4,863 * * *$ \\
(Einwohner/Fläche in $\left.\mathrm{km}^{2}\right)$ & & $(1,353)$ & $(1,438)$ \\
Dummies für Bundesländer & $\mathrm{Ja} * * *$ & $\mathrm{Ja} * * *$ & $\mathrm{Ja} * * *$ \\
Anzahl der Beobachtungen & 93 & 93 & 93 \\
$\mathrm{R}^{2}$ & 0,480 & 0,535 & 0,494 \\
\hline
\end{tabular}

Hinweise: Robuste Standardfehler in Klammern. ***: statistisch signifikant auf dem $0,1 \%$-Niveau; **: statistisch signifikant auf dem $1 \%$ Niveau; *: statistisch signifikant auf dem 5\%-Niveau.

lich des aktuellen Wohnorts, nicht aber in Bezug auf den Wohnort während der Jugendzeit festgestellt werden kann. Der Wert von Cohens d deutet allerdings darauf hin, dass dieser Unterschied als gering einzustufen ist. Der Anteil der Mittelwertunterschiede an der gemeinsamen Standardabweichung beträgt zirka 5,1 bzw. 4,6 \% (vgl. Tabelle 5).

Der stärker ausgeprägte Stadt-Land-Unterschied im Vorkommen unternehmerischer Persönlichkeitsprofile bezogen auf den aktuellen Wohnort deutet auf einen Einfluss von räumlicher Mobilität zwischen beiden Raumkategorien hin. Um dieser Vermutung nachzugehen, werden in den Spalten (e) bis (h) der Tabelle 5 die Persönlichkeitsprofile von Personen mit unterschiedlichen Mobilitätsmustern zwischen Stadt und Land miteinander verglichen. Eine Person in der Gruppe Stadt $\rightarrow$ Land (Spalte f) hat ihre Jugend in der Stadt verbracht, lebt zum Zeitpunkt der Befragung aber auf dem Land. Diese Gruppe wird mit Personen verglichen, die sowohl in ihrer Jugend als auch aktuell in der Stadt leben $($ Stadt $\rightarrow$ Stadt). Entsprechend steht die Gruppe Land $\rightarrow$ Stadt (Spalte h) für Personen, die ihre Jugend in ländlichen Regionen verbracht haben, aktuell aber in städtischen Regionen wohnen. Die Gruppe Land $\rightarrow$ Land (Spalte g) umfasst diejenigen Personen, die zur Jugendzeit und zum Zeitpunkt der Befragung in einer ländlichen Region gelebt haben.

Während sich der Unterschied bei den unternehmerischen Persönlichkeitsprofilen in den Gruppen Stadt $\rightarrow$ Stadt 
Abbildung 4 Regional unterschiedliche Verteilung der Erwerbsbevölkerung mit unternehmerischem Persönlichkeitsprofil in „Stadt“ und „Land“

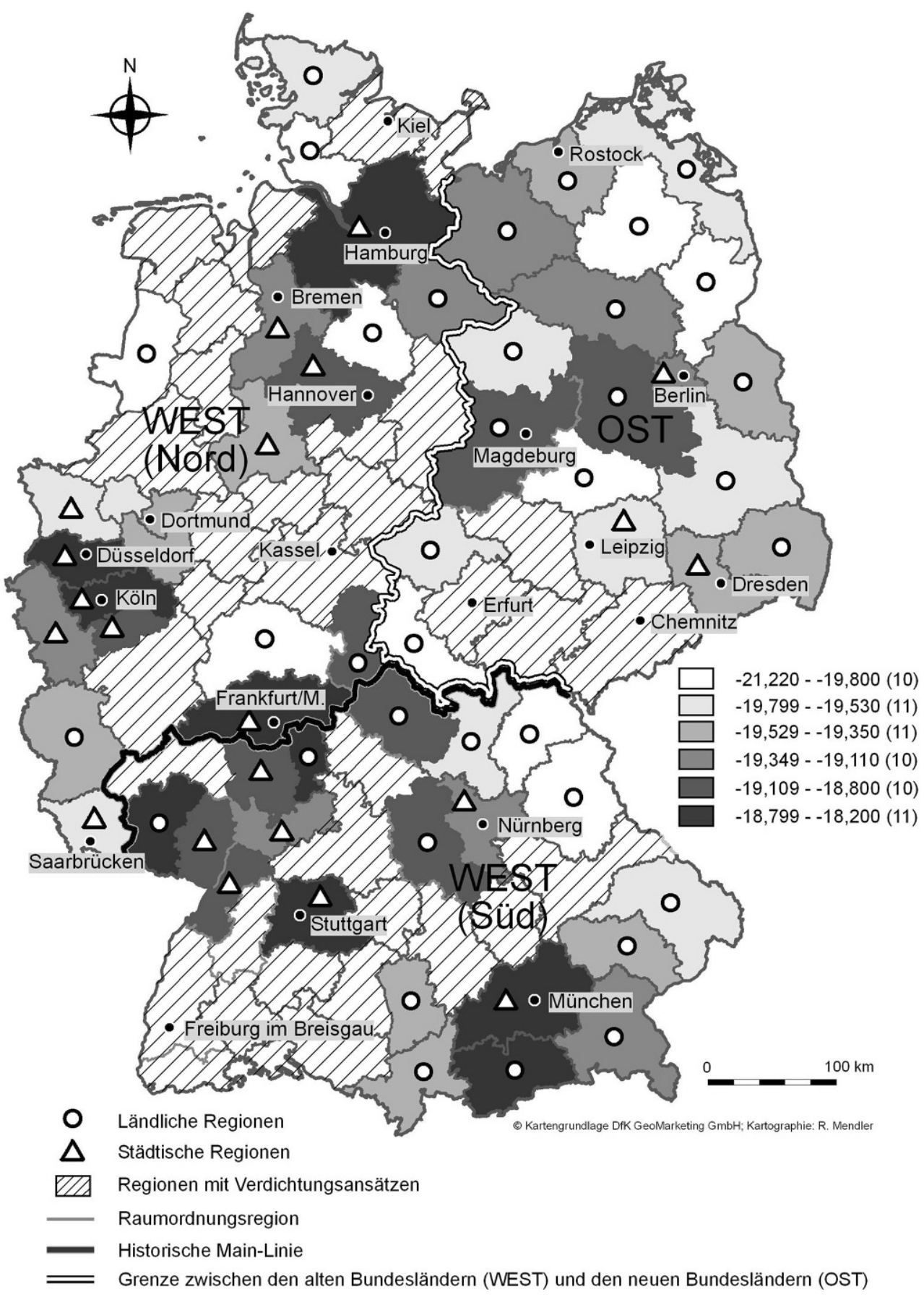

und Stadt $\rightarrow$ Land als nicht statistisch signifikant erweist, weist die Gruppe derjenigen Probanden, die vom Land in die Stadt gezogen sind (Land $\rightarrow$ Stadt) ein signifikant stärker unternehmerisch ausgeprägtes Persönlichkeitsprofil auf als die Gruppe derjenigen, die in einer ländlichen Region verblieben sind. Dies deutet darauf hin, dass Personen in ländlichen Regionen mit einer relativ stark unternehmerisch geprägten Persönlichkeitsstruktur eine besondere Tendenz haben, in eine städtische Region zu ziehen. Zwar zeigt der Wert für Cohens d eine nur geringe Effektstärke an, allerdings macht der Unterschied zwischen den Gruppen
Land $\rightarrow$ Land und Land $\rightarrow$ Stadt immerhin rund 5,7 \% der gemeinsamen Standardabweichung aus.

In einer weiteren Analyse beschränken wir den Vergleich auf die mobilen Bevölkerungsgruppen (Land $\rightarrow$ Stadt vs. Stadt $\rightarrow$ Land). Im Ergebnis zeigt sich, dass Personen, die in die Stadt abwandern, kein signifikant höheres unternehmerisches Profil aufweisen als Personen, die von der Stadt auf das Land umsiedeln (vgl. Tabelle 6). 
Tabelle 5 Stadt-Land-Unterschiede bezüglich der Persönlichkeitsstruktur nach Herkunft und aktuellem Wohnort (gewichtete Ergebnisse)

\begin{tabular}{|c|c|c|c|c|c|c|c|c|}
\hline & \multicolumn{2}{|c|}{ Wohnort Jugend } & \multicolumn{2}{|c|}{ Aktueller Wohnort } & \multicolumn{4}{|c|}{ Wohnort Jugend $\rightarrow$ Aktuell } \\
\hline & $\begin{array}{l}\text { (a) Stadt } \\
\text { (N=34.888) }\end{array}$ & $\begin{array}{l}\text { (b) Land } \\
\text { (N=15.976) }\end{array}$ & $\begin{array}{l}\text { (c) Stadt } \\
(\mathrm{N}=35.366)\end{array}$ & $\begin{array}{l}\text { (d) Land } \\
(\mathrm{N}=10.293)\end{array}$ & $\begin{array}{l}(\mathrm{e}) \\
\text { Stadt } \rightarrow \text { Stadt } \\
(\mathrm{N}=30.336)\end{array}$ & $\begin{array}{l}(\mathrm{f}) \\
\text { Stadt } \rightarrow \text { Land } \\
(\mathrm{N}=1.542)\end{array}$ & $\begin{array}{l}(\mathrm{g}) \\
\text { Land } \rightarrow \text { Land } \\
(\mathrm{N}=8.751)\end{array}$ & $\begin{array}{l}(\mathrm{h}) \\
\text { Land } \rightarrow \text { Stadt } \\
(\mathrm{N}=5.030)\end{array}$ \\
\hline Mittelwert & $-18,468$ & $-18,826$ & $-18,523$ & $-18,839$ & $-18,518$ & $-18,251$ & $-18,956$ & $-18,562$ \\
\hline Standardabweichung & 6,919 & 6,870 & 6,909 & 6,964 & 6,940 & 6,772 & 6,996 & 6,677 \\
\hline Statistische Signifikanz & $* * *(\mathrm{n} . \mathrm{s})$. & & $* *(* * *)$ & & n.s. (n.s.) & & $* *(* * *)$ & \\
\hline Cohens d & 0,052 & & 0,046 & & 0,039 & & 0,057 & \\
\hline $\begin{array}{l}\text { Anteil der Mittelwert- } \\
\text { unterschiede an der } \\
\text { gemeinsamen Stan- } \\
\text { dardabweichung }(\%)\end{array}$ & 5,181 & & 4,563 & & 3,851 & & 5,720 & \\
\hline
\end{tabular}

Anmerkungen: ***: statistisch signifikant auf dem 0,1\%-Niveau; **: statistisch signifikant auf dem 1\%-Niveau; *: statistisch signifikant auf dem 5\%-Niveau; n.s.: Unterschied nicht statistisch signifikant. Die statistische Signifikanz von Unterschieden der entsprechenden Standardabweichungen ist jeweils in Klammern angegeben. Der Test auf statistische Signifikanz für die Unterschiede bezüglich der Standardabweichungen kann nur auf Basis ungewichteter Werte durchgeführt werden.

Tabelle 6 Mobilität und Persönlichkeitsstruktur (gewichtete Ergebnisse)

\begin{tabular}{|c|c|c|c|c|c|c|}
\hline & \multicolumn{6}{|c|}{ Wohnort Jugend $\rightarrow$ aktuell } \\
\hline & Stadt/Land & & Ost/West & & Nord/Süd & \\
\hline & $\begin{array}{l}(\mathrm{f}) \text { Stadt } \rightarrow \text { Land } \\
(\mathrm{N}=1.542)\end{array}$ & $\begin{array}{l}(\mathrm{h}) \text { Land } \rightarrow \text { Stadt } \\
(\mathrm{N}=5.030)\end{array}$ & $\begin{array}{l}(f) \text { Ost } \rightarrow \text { West } \\
(\mathrm{N}=3.326)\end{array}$ & $\begin{array}{l}\text { (h) West } \rightarrow \text { Ost } \\
(\mathrm{N}=1.063)\end{array}$ & $\begin{array}{l}\text { (f) Nord } \rightarrow \text { Süd } \\
(\mathrm{N}=2.653)\end{array}$ & $\begin{array}{l}\text { (h) Süd } \rightarrow \text { Nord } \\
(\mathrm{N}=2.431)\end{array}$ \\
\hline Mittelwert & $-18,251$ & $-18,562$ & $-18,840$ & $-18,504$ & $-17,657$ & $-18,294$ \\
\hline Standardabweichung & 6,772 & 6,677 & 6,703 & 7,052 & 6,541 & 6,907 \\
\hline Statistische Signifikanz & n.s. (n.s.) & & n.s. (n.s.) & & $*(\mathrm{n} . \mathrm{s})$. & \\
\hline Cohens d & 0,046 & & 0,05 & & 0,095 & \\
\hline $\begin{array}{l}\text { Anteil der Mittelwertunterschiede an der } \\
\text { gemeinsamen Standardabweichung }(\%)\end{array}$ & 4,640 & & 4,962 & & 9,499 & \\
\hline
\end{tabular}

Anmerkungen: ***: statistisch signifikant auf dem 0,1\%-Niveau; **: statistisch signifikant auf dem 1\%-Niveau; *: statistisch signifikant auf dem 5\%-Niveau; n.s.: Unterschied nicht statistisch signifikant. Die statistische Signifikanz von Unterschieden der entsprechenden Standardabweichungen ist jeweils in Klammern angegeben.

\subsection{Unterschiede zwischen Ost- und Westdeutschland}

Es ist zu vermuten, dass 40 Jahre Sozialismus unter dem DDR-Regime in Ostdeutschland deutliche Spuren in Bezug auf die unternehmerische Einstellung der Bevölkerung hinterlassen haben (Fritsch/Bublitz/Sorgner et al. 2014; Wyrwich 2013). Dabei dürften zum einen die intensive Bekämpfung von privatem Unternehmertum etwa durch Verstaatlichung sowie die anti-kapitalistische Indoktrinierung der Bevölkerung (Fritsch/Rusakova 2012) eine Rolle gespielt haben. Zum anderen hat diese Politik gerade für Personen mit ausgeprägter unternehmerischer Einstellung einen besonderen Anreiz erzeugt, die DDR zu verlassen, sodass man vermuten kann, dass die unternehmerische Einstellung unter der verbliebenen Bevölkerung nur relativ schwach ausgeprägt ist.

Bei der Analyse der Unterschiede zwischen Ost- und Westdeutschland bleibt die Region Berlin unberücksichtigt, da der Ostteil und der Westteil der Stadt inzwischen so gut wie vollständig integriert sind, sodass eine Unterscheidung zwischen dem Westteil und dem ehemals zur DDR gehörenden Ostteil nicht sinnvoll ist. Unterscheidet man die Probanden danach, ob ihr Wohnort während der Ju- gend in Ost- oder Westdeutschland lag, so zeigt der Gesamtindikator zwar eine geringere Abweichung vom unternehmerischen Persönlichkeitsprofil für Westdeutschland an (Spalten a und b in Tabelle 7), allerdings ist dieser Unterschied nicht statistisch signifikant. Legt man der Auswertung von Ost-West-Unterschieden hingegen den aktuellen Wohnort zugrunde, so erweist sich der Unterschied zwischen Ost- und Westdeutschland als statistisch signifikant (Spalten c und d in Tabelle 7). Wie bei den Analysen von Stadt-Land-Unterschieden legen die divergierenden Ergebnisse für den Wohnort in der Jugendzeit und dem aktuellen Wohnort einen Einfluss der räumlichen Mobilität der Probanden nahe.

Wir finden heraus, dass Personen, die von Ostdeutschland nach Westdeutschland gewandert sind (Spalte $\mathrm{f}$ in Tabelle 7), eine geringere Abweichung vom unternehmerischen Idealprofil aufweisen als Personen, die in Ostdeutschland verblieben sind. Gemessen an Cohens d und dem Anteil an der gemeinsamen Standardabweichung ist dieser Effekt vergleichsweise stark ausgeprägt. In diesem Zusammenhang ist bemerkenswert, dass Personen, die von Westnach Ostdeutschland gewandert sind, ein signifikant weniger unternehmerisch ausgeprägtes Profil aufweisen als Personen, die in Westdeutschland verblieben sind (Spalten $g$ 
Tabelle 7 Ost-West-Unterschiede bezüglich der Ausprägung des unternehmerischen Persönlichkeitsprofils nach Herkunft und aktuellem Wohnort (gewichtete Ergebnisse)

\begin{tabular}{|c|c|c|c|c|c|c|c|c|}
\hline & \multicolumn{2}{|c|}{ Wohnort Jugend } & \multicolumn{2}{|c|}{ Aktueller Wohnort } & \multicolumn{4}{|c|}{ Wohnort Jugend $\rightarrow$ aktuell } \\
\hline & $\begin{array}{l}\text { (a) Ost } \\
(\mathrm{N}=12.343)\end{array}$ & $\begin{array}{l}\text { (b) West } \\
(\mathrm{N}=54.985)\end{array}$ & $\begin{array}{l}\text { (c) Ost } \\
(\mathrm{N}=10.080)\end{array}$ & $\begin{array}{l}\text { (d) West } \\
(\mathrm{N}=57.248)\end{array}$ & $\begin{array}{l}(\mathrm{e}) \mathrm{Ost} \rightarrow \text { Ost } \\
(\mathrm{N}=9.017)\end{array}$ & $\begin{array}{l}(\mathrm{f}) \\
\text { Ost } \rightarrow \text { West } \\
(\mathrm{N}=3.326)\end{array}$ & $\begin{array}{l}(\mathrm{g}) \text { West } \rightarrow \text { West } \\
(\mathrm{N}=53.922)\end{array}$ & $\begin{array}{l}(\mathrm{h}) \\
\text { West } \rightarrow \text { Ost } \\
(\mathrm{N}=1.063)\end{array}$ \\
\hline Mittelwert & $-18,778$ & $-18,574$ & $-18,865$ & $-18,566$ & $-18,868$ & $-18,504$ & $-18,569$ & $-18,840$ \\
\hline Standardabweichung & 6,790 & 6,933 & 6,837 & 6,920 & 6,816 & 6,703 & 6,931 & 7,052 \\
\hline Statistische Signifikanz & n.s. (n.s.) & & $*($ n.s. $)$ & & n.s. $(* *)$ & & $*($ n.s. $)$ & \\
\hline Cohens d & 0,03 & & 0,043 & & 0,054 & & 0,039 & \\
\hline $\begin{array}{l}\text { Anteil der Mittelwert- } \\
\text { unterschiede an der ge- } \\
\text { meinsamen Standard- } \\
\text { abweichung }(\%)\end{array}$ & 2,961 & & 4,329 & & 5,362 & & 3,908 & \\
\hline
\end{tabular}

Tabelle 8 Nord-Süd-Unterschiede bezüglich der Verteilung von Persönlichkeitsstrukturen nach Herkunft und aktuellem Wohnort (gewichtete Ergebnisse)

\begin{tabular}{|c|c|c|c|c|c|c|c|c|}
\hline & \multicolumn{2}{|c|}{ Wohnort Jugend } & \multicolumn{2}{|c|}{ Aktueller Wohnort } & \multicolumn{4}{|c|}{ Wohnort Jugend $\rightarrow$ aktuell } \\
\hline & $\begin{array}{l}\text { (a) Nord } \\
\text { (N=34.122) }\end{array}$ & $\begin{array}{l}\text { (b) Süd } \\
(\mathrm{N}=20.863)\end{array}$ & $\begin{array}{l}\text { (c) Nord } \\
(\mathrm{N}=33.900)\end{array}$ & $\begin{array}{l}\text { (d) Süd } \\
(\mathrm{N}=21.085)\end{array}$ & $\begin{array}{l}(\mathrm{e}) \\
\text { Nord } \rightarrow \text { Nord } \\
(\mathrm{N}=31.469)\end{array}$ & $\begin{array}{l}(\mathrm{f}) \\
\text { Nord } \rightarrow \text { Süd } \\
(\mathrm{N}=2.653)\end{array}$ & $\begin{array}{l}(\mathrm{g}) \\
\text { Süd } \rightarrow \text { Süd } \\
(\mathrm{N}=18.432)\end{array}$ & $\begin{array}{l}\text { (h) } \\
\text { Süd } \rightarrow \text { Nord } \\
(\mathrm{N}=2.431)\end{array}$ \\
\hline Mittelwert & $-18,541$ & $-18,624$ & $-18,606$ & $-18,526$ & $-18,630$ & $-17,657$ & $-18,664$ & $-18,294$ \\
\hline Standardabweichung & 7,005 & 6,819 & 7,035 & 6,780 & 7,044 & 6,541 & 6,808 & 6,907 \\
\hline Statistische Signifikanz & n.s. $(* * *)$ & & n.s. $(* * *)$ & & $* * *(* * *)$ & & n.s. (n.s.) & \\
\hline Cohens d & 0,012 & & 0,012 & & 0,139 & & 0,054 & \\
\hline $\begin{array}{l}\text { Anteil der Mittelwertunter- } \\
\text { schiede an der gemeinsa- } \\
\text { men Standardabweichung } \\
(\%)\end{array}$ & 1,206 & & 1,164 & & 13,879 & & 5,413 & \\
\hline
\end{tabular}

Anmerkungen: ***: statistisch signifikant auf dem 0,1\%-Niveau; **: statistisch signifikant auf dem 1\%-Niveau; *: statistisch signifikant auf dem 5\%-Niveau; n.s.: Unterschied nicht statistisch signifikant. Die statistische Signifikanz von Unterschieden der entsprechenden Standardabweichungen ist jeweils in Klammern angegeben. Der Test auf statistische Signifikanz für die Unterschiede bezüglich der Standardabweichungen kann nur auf der Basis ungewichteter Werte durchgeführt werden.

und $\mathrm{h}$ in Tabelle 7). Entsprechend weisen Westdeutsche in Ostdeutschland auch ein deutlich geringer ausgeprägtes unternehmerisches Persönlichkeitsprofil auf als Ostdeutsche, die nach Westdeutschland gewandert sind (vgl. Tabelle 6).

\subsection{Nord-Süd-Unterschiede}

Zur Analyse von Besonderheiten zwischen Nord- und Süddeutschland ziehen wir die historische Mainlinie als Unterscheidungsmerkmal heran, die allgemein als kulturelle Grenze zwischen Nord- und Süddeutschland angesehen wird. ${ }^{21}$ Unter „Süd“ werden hier Raumordnungsregionen verstanden, die südlich der Mainlinie liegen. Raumord-

${ }_{21}$ Nach dem Neuen Brockhaus 1960 ist Süddeutschland „,der südlich der mitteldeutschen Gebirgsschwelle gelegene Teil Deutschlands, etwa die Länder Bayern, Baden-Württemberg, das südliche Rheinland-Pfalz und Hessen südlich des Mains. Er setzt sich aus dem gewöhnlich Südwestdeutschland bezeichneten Gebiet der Oberrheinischen Tiefebene und der anschließenden Stufenlandschaft, der Oberdeutschen Hochebene (südlich der Donau) und den deutschen Alpen zusammen." $\mathrm{Zu}-$ sätzlich haben wir danach unterschieden, ob das Gebiet nach 1866 nungsregionen nördlich des Mains werden als „Nord“ bezeichnet. Personen aus den neuen Bundesländern und Berlin bleiben unberücksichtigt, um eine Verzerrung der Ergebnisse durch spezielle Ost-West-Unterschiede zu vermeiden.

Die Auswertungen zeigen, dass Probanden in Süddeutschland ein etwas stärker unternehmerisch ausgeprägtes Persönlichkeitsprofil aufweisen als Probanden in Norddeutschland. Allerdings ist dieser Unterschied sowohl bei Unterscheidung nach Wohnort in der Jugendzeit als auch aktuellem Wohnort nicht statistisch signifikant. Ein statistisch hochsignifikanter Unterschied zeigt sich allerdings zwischen denjenigen Probanden, die von Nord nach Süd gezogen sind, und denjenigen, die im Norden verblieben sind (Spalten e und $\mathrm{f}$ in Tabelle 8). Auch diejenigen Personen, die von Süd nach Nord gewandert sind, weisen

Teil des Norddeutschen Bunds gewesen ist. Dementsprechend haben wir Frankfurt am Main dem Norden zugerechnet, obwohl es nach der Brockhaus-Definition auch vertretbar gewesen wäre, die Region um Frankfurt am Main dem Süden zuzuordnen. 
ein stärker unternehmerisch geprägtes Persönlichkeitsprofil auf als diejenigen, die im Süden verblieben sind (Spalten $\mathrm{g}$ und $\mathrm{h}$ in Tabelle 8). Allerdings ist die Effektstärke der Nord-Süd-Wanderungen sowohl gemessen mit Cohens $\mathrm{d}$ als auch mit dem Anteil der Mittelwertunterschiede an der gemeinsamen Standardabweichung deutlich stärker ausgeprägt als der Effekt der Mobilität in umgekehrter Richtung. Vergleicht man die beiden mobilen Bevölkerungsgruppen miteinander (Nord $\rightarrow$ Süd vs. Süd $\rightarrow$ Nord), so zeigt sich, dass Personen, die von Nord nach Süd gewandert sind, ein höheres unternehmerisches Profil aufweisen als Personen, die von Süd nach Nord umsiedelten (vgl. Tabelle 6).

\section{Zusammenfassung und Schlussfolgerungen}

Ausgehend von einem empirisch fundierten unternehmerischen Persönlichkeitsprofil haben wir analysiert, inwieweit die Persönlichkeitsstruktur der Bevölkerung in den deutschen Raumordnungsregionen von diesem Idealbild abweicht. Dabei zeigen sich ganz erhebliche und statistisch signifikante Unterschiede, die durchaus mit einigen gängigen Stereotypen übereinstimmen.

Ebenso wie Analysen für Großbritannien und die USA (Obschonka/Schmitt-Rodermund/Silbereisen et al. 2013; Stuetzer/Obschonka/Audretsch et al. 2016) finden wir generell mehr unternehmerisch ausgeprägte Persönlichkeitsprofile in Verdichtungsgebieten. Abgesehen von diesem StadtLand-Unterschied ist ein relativ stark ausgeprägtes unternehmerisches Persönlichkeitsprofil in der Bevölkerung auch für die Regionen Westdeutschlands (im Vergleich zu Ostdeutschland) sowie innerhalb Westdeutschlands für Regionen südlich der Mainlinie feststellbar. Allerdings sind diese Effekte nur relativ schwach ausgeprägt und erklären nur einen relativ geringen Teil der beobachtbaren Streuung. Ein offensichtlicher Grund für diese geringe Erklärungskraft der Unterschiede nach Ost $\rightarrow$ West und Nord $\rightarrow$ Süd besteht in der stark ausgeprägten Streuung der unternehmerischen Persönlichkeitsprofile innerhalb dieser Raumkategorien.

Bei einem Abgleich der räumlichen Zuordnung der Probanden nach jetzigem Wohnort und dem Wohnort während der Jugendzeit zeigen sich einige bemerkenswerte Unterschiede, die auf die Bedeutung von räumlicher Mobilität hinweisen. Wir finden, dass Personen, die vom Land in die Stadt, von Ost- nach Westdeutschland sowie innerhalb Westdeutschlands von Nord nach Süd gezogen sind, in stärkerem Maße eine unternehmerische Persönlichkeitsstruktur aufweisen als Personen, die in die jeweils entgegengesetzte Richtung gewandert sind. Allerdings sind auch diese Effekte jeweils nur relativ schwach ausgeprägt. In vertiefenden Analysen ist zu klären, inwiefern die regionalen
Unterschiede im Vorhandensein unternehmerischer Persönlichkeitsstrukturen in Deutschland auf selektiven Wanderungen - bestimmte Typen von Personen werden von bestimmten Regionen angezogen bzw. abgestoßen - oder auf Sozialisationseffekten der Zugezogenen beruhen (vgl. auch Rentfrow/Gosling/Potter 2008; Obschonka/Schmitt-Rodermund/Silbereisen et al. 2013). ${ }^{22}$

Unsere Analysen werfen eine ganze Reihe wichtiger Fragen auf, die im Rahmen zukünftiger Untersuchungen geklärt werden sollten. Eine dieser Fragen betrifft die Auswirkungen von mehr oder weniger stark unternehmerisch geprägten Persönlichkeitsprofilen in der regionalen Bevölkerung auf die regionale Entwicklung in Deutschland, wie sie bereits durch Untersuchungen für Großbritannien und die USA belegt wurden. Führt eine stark ausgeprägt unternehmerische Einstellung der Bevölkerung einer Region zu einem hohen Niveau an Gründungsaktivität und der unternehmerischen Selbstständigkeit in dieser Region? Zeichnen sich Regionen mit relativ stark unternehmerisch geprägter Persönlichkeitsstruktur der Bevölkerung durch ein hohes Wohlstandsniveau und starkes Wachstum aus? Weiterhin sollte mehr über die Gründe für die regionalen Unterschiede der Ausprägung von Persönlichkeitsstrukturen bekannt sein. Wieso ist die Einstellung der Bevölkerung in einigen Regionen Deutschlands unternehmerischer ausgeprägt als in anderen Regionen? Welche Rolle spielen hierbei die Wirtschaftsgeschichte und insbesondere die regionale Wirtschaftsstruktur, wie beispielsweise der Anteil typischer Großindustrien? Sind Regionen mit relativ stark unternehmerisch geprägter Persönlichkeitsstruktur der Bevölkerung durch ein hohes Maß an unternehmerischer Selbstständigkeit in der Vergangenheit gekennzeichnet? Falls ja, wie kann dies historisch hohe Niveau regionaler unternehmerischer Selbstständigkeit erklärt werden? Inwiefern stehen Unterschiede im Vorhandensein von Persönlichkeitsstrukturen mit anderen Faktoren wie etwa institutionellen Regelungen (z. B. Erbschaftsrecht) ${ }^{23}$ in Zusammenhang?

\footnotetext{
22 Obschonka, Schmitt-Rodermund, Silbereisen et al. (2013: 109) vermuten auf der Grundlage einer Analyse für die USA, dass die besonders hohen Werte unternehmerischen Persönlichkeitsstrukturen im USamerikanischen Westen mit selektiver Einwanderung und Besiedlung von äußerst unternehmerisch eingestellten Personen (die das Abenteuer suchten und auch vor großen Strapazen und Gefahren nicht zurückschreckten) erklärt werden könnte.

${ }^{23}$ Eine Auswirkung des Erbschaftsrechts könnte darauf beruhen, dass bei einer Aufteilung der zu vererbenden landwirtschaftlichen Güter auf alle direkten Nachfahren (Realteilung) immer kleinere landwirtschaftliche Einheiten entstanden, die jeweils immer geringere Erträge abwarfen. Im Vergleich zu Regionen, in denen die landwirtschaftlichen Güter im Erbfall nicht aufgeteilt wurden (Anerbenrecht), entstand durch sinkende Einkommen ein Anreiz zu Aufnahme weiterer wirtschaftlicher Tätigkeit (etwa im Handwerk), der sich als förderlich für die Herausbildung von regionalem Unternehmertum erwies.
} 
In Verbindung mit den Gründen für regionale Unterschiede in der Persönlichkeitsstruktur der regionalen Bevölkerung wäre insbesondere die Bedeutung der räumlichen Mobilität näher zu analysieren. Inwiefern beruhen die beobachtbaren räumlichen Unterschiede in Deutschland auf der Mobilität von Personen? Was sind die präferierten Wanderungsziele von Personen mit unterschiedlich stark unternehmerisch geprägter Persönlichkeitsstruktur? Neigen Personen mit einer bestimmten (mehr oder weniger unternehmerisch geprägten) Persönlichkeitsstruktur dazu, in Regionen zu wandern, in denen die durchschnittliche Persönlichkeitsstruktur der Bevölkerung mit den eigenen Merkmalen übereinstimmt? Die Verfügbarkeit von psychologischen Landkarten mit Informationen über die Persönlichkeitsmerkmale der Bevölkerung ermöglicht eine Einbeziehung von wahrscheinlich wichtigen Faktoren in regionalen Analysen, über die bislang überwiegend nur spekuliert werden konnte (Saxenian 1994; Florida 2002). Die Ursachen für solche regionalen Unterschiede und ihr Stellenwert bei der Erklärung regionaler Entwicklung in Deutschland sind in weiteren Analysen zu klären.

\section{Literatur}

Audretsch, D. B.; Obschonka, M.; Gosling, S. D.; Potter, J. (2017). A new perspective on entrepreneurial regions: Linking cultural identity with latent and manifest entrepreneurship. In: Small Business Economics 48, 3, 681-697. doi: 10.1007/s11187-016-9787-9

Beesley, M. E.; Hamilton, R. T. (1984): Small Firms' Seedbed Role and the Concept of Turbulence. In: The Journal of Industrial Economics 33, 2, 217-231. doi: 10.2307/2098510

Benet-Martínez, V.; John, O. P. (1998): Los Cinco Grandes across cultures and ethnic groups: multitrait multimethod analyses of the Big Five in Spanish and English. In: Journal of Personality and Social Psychology 75, 3, 729-750.

Bersch, J.; Gottschalk, S.; Müller, B.; Niefert, M. (2014): The Mannheim Enterprise Panel (MUP) and Firm Statistics for Germany. Mannheim. = ZEW Discussion Paper 14-104.

Bleidorn, W.; Klimstra, T. .; Denissen, J. J.; Rentfrow, P. J.; Potter, J.; Gosling, S.D. (2013): Personality maturation around the world. A cross-cultural examination of social-investment theory. In: Psychological Science 24, 12, 2530-2540. doi: 10.1177/ 0956797613498396

Bortz, J.; Schuster, C. (2010): Statistik für Sozial- und Humanwissenschaftler. Heidelberg.

Bosma, N.; Hessels, J.; Schutjens, V.; van Praag, M.; Verheul, I. (2012): Entrepreneurship and role models. In: Journal of Economic Psychology 33, 2, 410-424. doi: 10.1016/j.joep.2011.03.004

Bosma, N.; Sternberg, R. (2014): Entrepreneurship as an Urban Event? Empirical Evidence from European Cities. In: Regional Studies 48, 6, 1016-1033. doi: 10.1080/00343404.2014.904041

Caliendo, M.; Fossen, F.; Kritikos, A. S. (2014): Personality characteristics and the decision to become and stay self-employed. In: Small Business Economics 42, 4, 787-814. doi: 10.1007/s11187013-9514-8

Chell, E. (2008): The entrepreneurial personality: A social construction. Abingdon.

Chinitz, B. (1961): Contrasts in Agglomeration: New York and Pittsburgh. In: American Economic Review 51, 2, 279-289.

Cohen, J. (1988): Statistical Power Analysis for the Behavioral Sciences. Hillsdale.
Digman, J. M. (1990): Personality structure: Emergence of the fivefactor model. In: Annual Review of Psychology 41, 1, 417-440. doi: 10.1146/annurev.ps.41.020190.002221

Elliott, M. R. (2008): Model Averaging Methods for Weight Trimming. In: Journal of Official Statistics 24, 4, 517-540.

Falck, O.; Heblich, S.; Lameli, A.; Südekum, J. (2012): Dialects, cultural identity, and economic exchange. In: Journal of Urban Economics 72, 2-3, 225-239. doi: 10.1016/j.jue.2012.05.007

Florida, R. (2002): The Rise of the Creative Class and how it's Transforming Work, Leisure, Community and Everyday Life. New York.

Florida, R.; Adler, P.; Mellander, S. (2017): The city as innovation machine. In: Regional Studies 51, 1, 86-96. doi: 10.1080/00343404. 2016.1255324

Fritsch, M.; Bublitz, E.; Sorgner, A.; Wyrwich, M. (2014): How much of a socialist legacy? The re-emergence of entrepreneurship in the East German transformation to a market economy. In: Small Business Economics 43, 2, 427-446. doi: 10.1007/s11187-0149544-X

Fritsch, M.; Rusakova, A. (2012): Self-Employment after Socialism: Intergenerational Links, Entrepreneurial Values, and Human Capital. In: International Journal of Developmental Science 6, 3-4, 167-175. doi: 10.3233/DEV-2012-12106

Fritsch, M.; Wyrwich, M. (2014): The Long Persistence of Regional Levels of Entrepreneurship: Germany 1925-2005. In: Regional Studies 48, 6, 955-973. doi: https://doi.org/10.1080/00343404. 2013.816414

Fritsch, M.; Wyrwich, M. (2017): Persistence of Regional Entrepreneurship: Causes, Effects, and Directions for Future Research. In: International Review of Entrepreneurship 15, 4, 395-416.

Glaeser, E. L. (2011a): Cities, productivity, and quality of life. In: Science 333, 6042, 592-594. doi: 10.1126/science.1209264

Glaeser, E. L. (2011b): Triumph of the city: How our greatest invention makes us richer, smarter, greener, healthier and happier. London.

Glaeser, E. L.; Kerr, S. P.; Kerr, W. R. (2015): Entrepreneurship and urban growth: An empirical assessment with historical mines. In: The Review of Economics and Statistics 97, 2, 498-520.

Gruhl, A.; Schmucker, A.; Seth, S. (2012): Das Betriebs-Historik-Panel 1975-2010* Handbuch Version 2.1.1. Nürnberg. = FDZ-Datenreport 04/2012 (de).

Hartke, W. (1959): Gedanken über die Bestimmung von Räumen gleichen sozialgeographischen Verhaltens. In: Erdkunde 13, 4, 426436. doi: 10.3112/erdkunde.1959.04.13

Helsley, R. .; Strange, W. C. (2011): Entrepreneurs and cities: Complexity, thickness and balance. In: Regional Science and Urban Economics 41, 6, 550-559. doi: 10.1016/j.regsciurbeco.2011.04.001

Horvitz, D. G.; Thompson, D. J. (1952): A Generalization of Sampling Without Replacement from a Finite Universe. In: Journal of the American Statistical Association 47, 260, 663-685.

Isaacson, W. (2011): Steve Jobs. New York.

John, O. P.; Srivastava, S. (1999): The Big Five trait taxonomy: History, measurement, and theoretical perspectives. In: Pervin, L. A.; John, O. P. (Hrsg.): Handbook of personality: Theory and research. New York, 102-138.

Loehlin, J. C. (1992): Genes and environment in personality development. Newbury Park, CA.

Loehlin, J. C.; McCrae, R. R.; Costa, P. T. Jr.; John, O. P. (1998): Heritabilities of common and measure-specific components of the Big Five personality factors. In: Journal of Research in Personality 32, 4, 431-453. doi: 10.1006/jrpe.1998.2225

Magnusson, D.; Törestad, B. (1993): A holistic view of personality: A model revisited. In: Annual Review of Psychology 44, 427-452. doi: 10.1146/annurev.ps.44.020193.002235

McCrae, R. R.; Costa, P. T. Jr. (1999): A five-factor theory of personality. In: Pervin, L. A.; John, O. P. (Hrsg.): Handbook of personality: Theory and research. New York, 139-153. 
Miegel, M. (1991): Wirtschafts- und arbeitskulturelle Unterschiede in Deutschland. Zur Wirkung außerökonomischer Faktoren auf die Beschäftigung. Gütersloh.

Obschonka, M.; Schmitt-Rodermund, E.; Silbereisen, R. K.; Gosling, S. D.; Potter, J. (2013): The regional distribution and correlates of an entrepreneurship-prone personality profile in the United States, Germany, and the United Kingdom: A socioecological perspective. In: Journal of Personality and Social Psychology 105, 1, 104122. doi: $10.1037 / \mathrm{a} 0032275$

Obschonka, M.; Stuetzer, M. (2017): Integrating Psychological Approaches to Entrepreneurship: The Entrepreneurial Personality System (EPS). In: Small Business Economics 49, 1, 203-231. doi: 10.1007/s11187-016-9821-y

Obschonka, M.; Stuetzer, M.; Audretsch, D. B.; Rentfrow, P. J.; Potter, J.; Gosling, S. D. (2016): Macropsychological factors predict regional economic resilience during a major economic crisis. In: Social Psychological and Personality Science 7, 2, 95-104. doi: 10.1177/1948550615608402

Obschonka, M.; Stuetzer, M.; Gosling, S. D.; Rentfrow, P. J.; Lamb, M. E.; Potter, J.; Audretsch, D. B. (2015): Entrepreneurial regions: Do macro-psychological cultural characteristics of regions help solve the "knowledge paradox" of economics? In: Plos one 10, 6, e012933. doi: 10.1371/journal.pone.0129332

Obschonka, M.; Stuetzer, M.; Rentfrow, P. J.; Potter, J.; Gosling, S. D. (2017): Did strategic bombing in the Second World War lead to 'German Angst'? A large-scale empirical test across 89 German cities. In: European Journal of Personality 31, 3, 234-257. doi: 10.1002/per.2104

Parker, S. (2009): Why do small firms produce the entrepreneurs? In: The Journal of Socio-Economics 38, 3, 484-494.

Rauch, A.; Frese, M. (2007): Let's put the person back into entrepreneurship research. A meta-analysis on the relationship between business owners' personality traits, business creation, and success. In: European Journal of Work and Organizational Psychology 16, 4, 353-385. doi: 10.1080/13594320701595438
Rentfrow, P. J.; Gosling, S. D.; Potter, J. (2008): A theory of the emergence, persistence, and expression of geographic variation in psychological characteristics. In: Perspectives on Psychological Science 3, 5, 339-369. doi: 10.1111/j.1745-6924.2008.00084.x

Roberts, B. W.; Walton, K. E.; Viechtbauer, W. (2006): Patterns of mean-level change in personality traits across the life course: a meta-analysis of longitudinal studies. In: Psychological Bulletin $132,1,1-25$.

Saxenian, A. (1994): Regional Advantage: Culture and Competition in Silicon Valley and Route 128. Cambridge, MA.

Schumpeter, J. A. (1934): The theory of economic development. Cambridge, MA.

Shane, S.; Nicolaou, N.; Cherkas, L.; Spector, T. D. (2010): Genetics, the Big Five, and the tendency to be self-employed. In: Journal of Applied Psychology 95, 6, 1154-1162. doi: 10.1037/a0020294

Siart, O.; Lang, F. (2016) (Hrsg.): Die Schwaben. Zwischen Mythos und Marke. Stuttgart.

Sorenson, O. (2017): Regional ecologies of entrepreneurship. In: Journal of Economic Geography 17, 5, 959-974. doi: 10.1093/jeg/ $\operatorname{lbx} 031$

Stuetzer, M.; Audretsch, D. B.; Obschonka, M.; Gosling, S. D.; Rentfrow, P. J.; Potter, J. (2017): Entrepreneurship culture, knowledge spillovers and the growth of regions. In: Regional Studies. doi: $10.1080 / 00343404.2017 .1294251$

Stuetzer, M.; Obschonka, M.; Audretsch, D. B.; Wyrwich, M.; Rentfrow, P. J.; Coombes, M.; Satchell, M. (2016): Industry structure, entrepreneurship, and culture: An empirical analysis using historical coalfields. In: European Economic Review 86, 52-72. doi: 10.1016/j.euroecorev.2015.08.012

Wyrwich, M. (2013): Can socioeconomic heritage produce a lost generation with regard to entrepreneurship? In: Journal of Business Venturing 28, 5, 667-682. doi.org/10.1016/j.jbusvent.2012.09.001

Zhao, H.; Seibert, S. E.; Lumpkin, G. T. (2010): The Relationship of Personality to Entrepreneurial Intentions and Performance: A Meta-Analytic Review. In: Journal of Management 36, 2, 381-404. doi: $10.1177 / 0149206309335187$ 